\title{
Deciding Whether a Regular Language is Generated by a Splicing System
}

\author{
Lila Kari Steffen Kopecki
}

\author{
Department of Computer Science \\ The University of Western Ontario \\ Middlesex College, London ON N6A 5B7 Canada \\ $\{$ lila, steffen\}@csd.uwo.ca
}

\begin{abstract}
Splicing as a binary word/language operation is inspired by the DNA recombination under the action of restriction enzymes and ligases, and was first introduced by Tom Head in 1987. Shortly thereafter, it was proven that the languages generated by (finite) splicing systems form a proper subclass of the class of regular languages. However, the question of whether or not one can decide if a given regular language is generated by a splicing system remained open. In this paper we give a positive answer to this question. Namely, we prove that, if a language is generated by a splicing system, then it is also generated by a splicing system whose size is a function of the size of the syntactic monoid of the input language, and which can be effectively constructed.
\end{abstract}

\section{Introduction}

In [10] Head described an language-theoretic operation, called splicing, which models DNA recombination, a cut-and-paste operation on DNA double-strands. Recall that a DNA single-strand is a polymer consisting of a series of the nucleobases Adenine (A), Cytosine (C), Guanine (G), and Thymine ( $\mathrm{T}$ ) attached to a linear, directed backbone. Due to the chemical structure of the backbone, the ends of a single-strand are called $3^{\prime}$-end and 5'-end. Abstractly, a DNA single-strand can be viewed as a string over the four letter alphabet $\{A, C, G, T\}$. The bases $A$ and $T$, respectively $C$ and $\mathrm{G}$, are Watson-Crick-complementary, or simply complementary, which means they can attach to each other via hydrogen bonds. The complement of a DNA single-strand $\alpha=5^{\prime}-a_{1} \cdots a_{n}-3^{\prime}$ is the strand $\bar{\alpha}=3^{\prime}-\overline{a_{1}} \ldots \overline{a_{n}}-5^{\prime}$ where $a_{1}, \ldots, a_{n}$ are bases and $\overline{a_{1}}, \ldots, \overline{a_{n}}$ denote their complementary bases, respectively; note that $\alpha$ and $\bar{\alpha}$ have opposite orientation. A strand $\alpha$ and its complement $\bar{\alpha}$ can bond to each other to form a DNA (double-)strand.

Splicing is meant to abstract the action of two compatible restriction enzymes and the ligase enzyme on two DNA double-strands. The first restriction enzyme recognizes a base-sequence $u_{1} v_{1}$, called its restriction site, in any DNA string, and cuts the string containing this factor between $u_{1}$ and $v_{1}$. The second restriction enzyme, with restriction site $u_{2} v_{2}$, acts similarly. Assuming that the sticky ends obtained after these cuts are complementary, the enzyme ligase aids then the recombination (catenation) of the first segment of one cut string with the second segment of another cut string. For example, the enzyme TaqI has restriction site TCGA, and the enzyme SciNI has restriction site GCGC. The enzymes cut double-strands

$$
\begin{array}{ll}
5^{\prime}-\alpha-\mathrm{TCGA}-\beta-3^{\prime} & 5^{\prime}-\gamma-\mathrm{GCGC}-\delta-3^{\prime} \\
3^{\prime}-\bar{\alpha}-\mathrm{AGCT}-\bar{\beta}-5^{\prime} & \text { and } \quad 3^{\prime}-\bar{\gamma}-\mathrm{CGCG}-\bar{\delta}-5^{\prime}
\end{array}
$$

along the dotted lines, respectively, leaving the first segment of the left strand with a sticky end GC which is compatible to the sticky end CG of the second segment of the right strand. The 
segments can be recombined to form either the original strands or the new strand

$$
\begin{aligned}
& 5^{\prime}-\alpha-\mathrm{T} \text { C G C }-\delta-3^{\prime} \\
& 3^{\prime}-\bar{\alpha}-\mathrm{A} \text { G C G }-\bar{\delta}-5^{\prime} .
\end{aligned}
$$

A splicing system is a formal language model which consists of a set of initial words or axioms I and a set of splicing rules $R$. The most commonly used definition for a splicing rule is a quadruple of words $r=\left(u_{1}, v_{1} ; u_{2}, v_{2}\right)$. This rule splices two words $x_{1} u_{1} v_{1} y_{1}$ and $x_{2} u_{2} v_{2} y_{2}$ : the words are cut between the factors $u_{1}, v_{1}$, respectively $u_{2}, v_{2}$, and the prefix (the left segment) of the first word is recombined by catenation with the suffix (the right segment) of the second word, see Figure 1 and also [18]. A splicing system generates a language which contains every word that can be obtained by successively applying rules to axioms and the intermediately produced words.

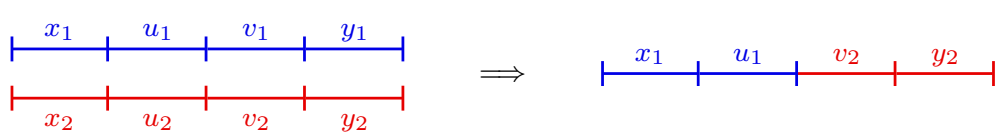

Figure 1: Splicing of the words $x_{1} u_{1} v_{1} y_{1}$ and $x_{2} u_{2} v_{2} y_{2}$ by the rule $r=\left(u_{1}, v_{1} ; u_{2}, v_{2}\right)$.

Example 1.1. Consider the splicing system $(I, R)$ with axiom $I=\{a b\}$ and rules $R=\{r, s\}$ where $r=(a, b ; \varepsilon, a b)$ and $s=(a b, \varepsilon ; a, b)$; in this paper, $\varepsilon$ denotes the empty word. Applying the rule $r$ to two copies of the axiom $a b$ creates the word $a a b$ and applying the rule $s$ to two copies of the axiom $a b$ creates the word $a b b$. More generally, the rule $r$ or $s$ can be applied to words $a^{i} b^{j}$ and $a^{k} b^{\ell}$ with $i, j, k, \ell \geq 1$ in order to create the word $a^{i+1} b^{\ell}$ or $a^{i} b^{\ell+1}$, respectively. The language generated by the splicing system $(I, R)$ is $L(I, R)=a^{+} b^{+}$.

The most natural variant of splicing systems, often referred to as finite splicing systems, is to consider a finite set of axioms and a finite set of rules. In this paper, by a splicing system we always mean a finite splicing system. Shortly after the introduction of splicing in formal language theory, Culik II and Harju [6] proved that splicing systems generate regular languages, only; see also $[12,17]$. Gatterdam [7] gave $(a a)^{*}$ as an example of a regular language which cannot be generated by a splicing system; thus, the class of languages generated by splicing systems is strictly included in the class of regular languages. However, for any regular language $L$ over an alphabet $\Sigma$, adding a marker $b \notin \Sigma$ to the left side of every word in $L$ results in the language $b L$ which can be generated by a splicing system [11]; e.g., the language $b(a a)^{*}$ is generated by the axioms $\{b, b a a\}$ and the rule $(b a a, \varepsilon ; b, \varepsilon)$.

This led to the question of whether or not one of the known subclasses of the regular languages corresponds to the class $\mathcal{S}$ of languages which can be generated by a splicing system. All investigations to date indicate that the class $\mathcal{S}$ does not coincide with another naturally defined language class. A characterization of reflexive splicing systems using Schützenberger constants has been given by Bonizzoni, de Felice, and Zizza [1-3]. A splicing system is reflexive if for all rules $\left(u_{1}, v_{1} ; u_{2}, v_{2}\right)$ in the system we have that $\left(u_{1}, v_{1} ; u_{1}, v_{1}\right)$ and $\left(u_{2}, v_{2} ; u_{2}, v_{2}\right)$ are rules in the system, too. A word $v$ is a Schützenberger constant of a language $L$ if $x_{1} v y_{1} \in L$ and $x_{2} v y_{2} \in L$ imply $x_{1} v y_{2} \in L$ [19]. Recently, it was proven by Bonizzoni and Jonoska that every splicing language has a constant [5]. However, not all languages which have a constant are generated by splicing systems, e. g., in the language $L=(a a)^{*}+b^{*}$ every word $b^{i}$ is a constant, but $L$ is not generated by a splicing system.

Another approach was to find an algorithm which decides whether a given regular language is generated by a splicing system. This problem has been investigated by Goode, Head, and Pixton $[8,9,13]$ but it has only been partially solved: it is decidable whether a regular language is generated by a reflexive splicing system. It is worth mentioning that a splicing system by the original definition in [10] is always reflexive. A related problem has been investigated by Kim [16]: given a regular language $L$ and a finite set of enzymes, represented by set of reflexive rules $R, \mathrm{Kim}$ showed that it is decidable whether or not $L$ can be generated from a finite set of axioms by using only rules from $R$. 
In this paper we settle the decidability problem, by proving that for a given regular language, it is indeed decidable whether the language is generated by a splicing system (which is not necessarily reflexive), Corollary 5.2. More precisely, for every regular language $L$ there exists a splicing system $\left(I_{L}, R_{L}\right)$ and if $L$ is a splicing language, then $L$ is generated by the splicing system $\left(I_{L}, R_{L}\right)$. The size of this splicing system depends on the size of the syntactic monoid of $L$. If $m$ is the size of the syntactic monoid of $L$, then all axioms in $I_{L}$ and the four components of every rule in $R_{L}$ have length in $\mathcal{O}\left(\mathrm{m}^{2}\right)$, Theorem 4.1. By results from [12,13], we can construct a finite automaton which accepts the language generated by $\left(I_{L}, R_{L}\right)$, compare it with a finite automaton which accepts $L$, and thus, decide whether $L$ is generated by a splicing system. Furthermore, we prove a similar result for a more general variant of splicing that has been introduced by Pixton [17], Theorem 3.1.

The paper is organized as follows. In Section 2 we lay down the notation, recall some wellknown results about syntactic monoids, and prove a pumping argument that is of importance for the proofs in the succeeding sections. Section 3 (Section 4) contains the proof that a regular language $L$ is generated by a Pixton splicing system (resp. classical splicing system) if and only if it is generated by one particular Pixton splicing system (resp. classical splicing system) whose sice is bounded by the size of the syntactic monoid of $L$. Sections 3 and 4 can be read independently and overlap in some of their main ideas. The inclusion of both sections and the presentation order are chiefly for expository purposes: Due to the features of the Pixton splicing, Section 3 introduces the main ideas in a significantly more readable way. Finally, in Section 5 we deduce the decidability results for both splicing variants.

An extended abstract of this paper, including a shortened proof of Theorem 4.1 and Corollary 5.2 i.), has been published in the conference proceedings of DNA 18 in 2012 [15]. Theorem 3.1 and Corollary 5.2 ii.) have not been published elsewhere.

\section{Notation and Preliminaries}

We assume the reader to be familiar with the fundamental concepts of language theory, see [14].

Let $\Sigma$ be a finite set of letters, the alphabet; $\Sigma^{*}$ be the set of all words over $\Sigma$; and $\varepsilon$ denote the empty word. A subset $L$ of $\Sigma^{*}$ is a language over $\Sigma$. Throughout this paper, we consider languages over the fixed alphabet $\Sigma$, only. Let $w \in \Sigma^{*}$ be a word. The length of $w$ is denoted by $|w|$. (We use the same notation for the cardinality $|S|$ of a set $S$, as usual.) We consider the letters of $\Sigma$ to be ordered and for words $u, v \in \Sigma^{*}$ we denote the length-lexicographical order by $u \leq_{\ell \ell} v$; i. e., $u \leq_{\ell \ell} v$ if either $|u| \leq|v|$, or $|u|=|v|$ and $u$ is at most $v$ in lexicographic order. The strict length-lexicographic order is denoted by $<_{\ell \ell}$; we have $u<_{\ell \ell} v$ if $u \leq_{\ell \ell} v$ and $u \neq v$.

For a length bound $m \in \mathbb{N}$ we let $\Sigma \leq m$ denote the set of words whose length is at most $m$, i. e., $\Sigma \leq m=\bigcup_{i \leq m} \Sigma^{i}$. Analogously, we define $\Sigma^{<m}=\bigcup_{i<m} \Sigma^{i}$.

If $w=x y z$ for some $x, y, z \in \Sigma^{*}$, then $x, y$, and $z$ are called prefix, factor, and suffix of $w$, respectively. If a prefix or suffix of $w$ is distinct from $w$, it is said to be proper.

Let $w=a_{1} \ldots a_{n}$ where $a_{1}, \ldots, a_{n}$ are letters from $\Sigma$. By $w[i]$ for $0 \leq i \leq n$ we denote a position in the word $w$ : if $i=0$, it is the position before the first letter $a_{1}$, if $i=n$ it is the position after the last letter $a_{n}$, and otherwise, it is the position between the letters $a_{i}$ and $a_{i+1}$. We want to stress that $w[i]$ is not a letter in the word $w$. By $w[i ; j]$ for $0 \leq i \leq j \leq n$ we denote the factor $a_{i+1} \cdots a_{j}$ which is enclosed by the positions $w[i]$ and $w[j]$. If $x=w[i ; j]$ we say the factor $x$ starts at position $w[i]$ and ends at position $w[j]$. Whenever we talk about a factor $x$ of a word $w$ we mean a factor starting (and ending) at a certain position, even if the the word $x$ occurs as a factor at several positions in $w$. Let $x=w[i ; j]$ and $y=w\left[i^{\prime} ; j^{\prime}\right]$ be factors of $w$. We say the factors $x$ and $y$ match (in $w$ ) if $i=i^{\prime}$ and $j=j^{\prime}$; the factor $x$ is covered by the factor $y$ (in $w$ ) if $i^{\prime} \leq i \leq j \leq j^{\prime}$; and the factors $x$ and $y$ overlap (in $w$ ) if $x \neq \varepsilon, y \neq \varepsilon$, and $i \leq i^{\prime}<j$ or $i^{\prime} \leq i<j^{\prime}$. In other words, if two factors $x$ and $y$ overlap in $w$, then they share a common letter of $w$. Let $x=w[i ; j]$ be a factor of $w$ and let $p=w[k]$ be a position in $w$. We say the position $p$ lies at the left of $x$ if $k \leq i$; the position $p$ lies at the right of $x$ if $k \geq j$; and the position $p$ lies in $x$ if $i<k<j$.

Every language $L$ induces an syntactic congruence $\sim_{L}$ over words such that $u \sim_{L} v$ if and only 
if for all words $x, y$

$$
x u y \in L \Longleftrightarrow x v y \in L .
$$

The syntactic class (with respect to $L$ ) of a word $u$ is $[u]_{L}=\left\{v \mid u \sim_{L} v\right\}$. The syntactic monoid of $L$ is the quotient monoid

$$
M_{L}=\Sigma^{*} / \sim_{L}=\left\{[u]_{L} \mid u \in \Sigma^{*}\right\} .
$$

It is well known that a language $L$ is regular if and only if its syntactic monoid $M_{L}$ is finite. We will use two basic facts about syntactic monoids of regular languages.

Lemma 2.1. Let $L$ be a regular language and let $w$ be a word with $|w| \geq\left|M_{L}\right|^{2}$. We can factorize $w=\alpha \beta \gamma$ with $\beta \neq \varepsilon$ such that $\alpha \sim_{L} \alpha \beta$ and $\gamma \sim_{L} \beta \gamma$.

Proof. Consider a word $w$ with $n=|w| \geq\left|M_{L}\right|^{2}$. For $i=0, \ldots, n$, let $X_{i}=w[0 ; i]$ be the syntactic classes of the prefixes of $w$ and let $Y_{i}=w[i ; n]$ be the syntactic classes of the suffixes of $w$. Note that $X_{i} Y_{i}=[w]_{L}$. By the pigeonhole principle, there are $i, j$ with $0 \leq i<j \leq n$ such that $X_{i}=X_{j}$ and $Y_{i}=Y_{j}$. Let $\alpha=w[0 ; i], \beta=w[i ; j]$, and $\gamma=w[j ; n]$. As $\alpha \in X_{i}$ and $\alpha \beta \in X_{j}$, we see that $\alpha \sim_{L} \alpha \beta$ and, symmetrically, $\gamma \sim_{L} \beta \gamma$.

Lemma 2.2. Let $L$ be a regular language. Every element $X \in M_{L}$ contains a word $x \in X$ with $|x|<\left|M_{L}\right|$.

Proof. We define a series of sets $S_{i} \subseteq M_{L}$. We start with $S_{0}=\{1\}$ (here, $1=[\varepsilon]_{L}$ ) and let $S_{i+1}=S_{i} \cup\left\{X \cdot[a]_{L} \mid X \in S_{i} \wedge a \in \Sigma\right\}$ for $i \geq 0$. It is not difficult to see that $X \in S_{i}$ if and only if $X$ contains a word $x \in X$ with $|x| \leq i$. As $S_{i} \subseteq S_{i+1}$ and $M_{L}$ is finite, the series has a fixed point $S_{n}$ such that $S_{i}=S_{n}$ for all $i \geq n$. Let $n$ be the least value with this property, i. e., $S_{n-1} \subsetneq S_{n}$ or $n=0$. Observe that $n<\left|M_{L}\right|$ as $S_{0} \subsetneq S_{1} \subsetneq \cdots \subsetneq S_{n}$. Every element $X \in M_{L}$ contains some word $w \in X$, thus, $X \in S_{|w|} \subseteq S_{n}$. Concluding that $X$ contains a word with a length of at most $n<\left|M_{L}\right|$.

\subsection{A Pumping Algorithm}

Consider a regular language $L$, a word $\alpha \beta \gamma$ where $\alpha \sim_{L} \alpha \beta$ and $\gamma \sim_{L} \beta \gamma$, due to Lemma 2.1, and a large even number $j$. In the proofs of Theorem 3.1 and Lemma 4.8, we need a pumping argument to replace all factors $\alpha \beta \gamma$ by $\alpha \beta^{j} \gamma$ in a word $z$ in order to obtain a word $\tilde{z}$; thus, $z \sim_{L} \tilde{z}$. As $\alpha \beta \gamma$ may be a factor of $\alpha \beta^{j} \gamma$, we cannot ensure that $\alpha \beta \gamma$ is not a factor of $\tilde{z}$. However, we can ensure that if $\alpha \beta \gamma=\tilde{z}\left[k ; k^{\prime}\right]$ is a factor of $\tilde{z}$, then either (a) $\alpha \beta^{j / 2}$ is a factor of $\tilde{z}$ starting at position $\tilde{z}[k]$ or (b) $\beta^{j / 2} \gamma$ is a factor of $\tilde{z}$ ending at position $\tilde{z}\left[k^{\prime}\right]$; i. e., either $\alpha$ is succeeded by a large number of $\beta$ 's or $\gamma$ is preceded by a large number of $\beta$ 's. The next lemma is a technical result whose purpose is to assure that for any word $z$ there exists a word $\tilde{z}$ such that the above-mentioned property holds and $\tilde{z}$ is generated by applying several successive pumping steps $\alpha \beta \gamma \mapsto \alpha \beta^{j} \gamma$ to $z$.

Lemma 2.3. Let $z, \alpha, \beta, \gamma$ be words with $\beta \neq \varepsilon$, let $\ell=|\alpha \beta \gamma|$, and let $j>|z|+\ell$ be an even number. The following algorithm will terminate and output $\tilde{z}$.

1. $\tilde{z}:=z$;

2. if $\tilde{z}[k ; k+\ell]=\alpha \beta \gamma$ for some $k$ such that neither

(a) $\alpha \beta^{j / 2}$ is a factor of $\tilde{z}$ starting at position $\tilde{z}[k]$ nor

(b) $\beta^{j / 2} \gamma$ is a factor of $\tilde{z}$ ending at position $\tilde{z}[k+\ell]$,

then let $\tilde{z}:=\tilde{z}[0 ; k] \cdot \alpha \beta^{j} \gamma \cdot \tilde{z}[k+\ell ;|\tilde{z}|] ;\left(\right.$ replace the factor $\tilde{z}[k ; k+\ell]=\alpha \beta \gamma$ in $\tilde{z}$ by $\left.\alpha \beta^{j} \gamma\right)$

3. repeat step 2 until there is no such factor $\alpha \beta \gamma$ in $\tilde{z}$ left. 
Before we prove Lemma 2.3, let us recall a basic fact about primitive words. A word $p$ is called primitive if there is no word $x$ and $i \geq 2$ such that $p=x^{i}$. The primitive root of a word $w \neq \varepsilon$ is the unique primitive word $p$ such that $w=p^{i}$ for some $i \geq 1$. For primitive $p$, it is well known that if $p p=x p y$, then either $x=p$ and $y=\varepsilon$, or $x=\varepsilon$ and $y=p$. In other words, whenever $p$ is a factor of $p^{n}$ starting at position $p^{n}[i]$, then $i \in|p| \cdot \mathbb{N}$.

For a word $w=x y$ we employ the notations $x^{-1} w=y$ and $w y^{-1}=x$. If $x$ is not a prefix of $w(y$ is not a suffix of $w)$, then the $x^{-1} w\left(\right.$ resp. $\left.w y^{-1}\right)$ is undefined.

Proof of Lemma 2.3. Let $p$ be the primitive root of $\beta$ and let $m$ such that $\beta=p^{m}$.

First, observe that if, during the computation, a factor $\alpha \beta \gamma=\tilde{z}[k ; k+\ell]$ is covered by a factor $\alpha \beta^{j} \gamma$ in $\tilde{z}$, then either (a) or (b) holds. Indeed, if $\alpha \beta \gamma=\left(\alpha \beta^{j} \gamma\right)[i ; i+\ell]$ for some $i$, then $\beta$ is a factor of $\beta^{j}$ starting at position $\beta^{j}[i]$. As mentioned above, $i \in|p| \cdot \mathbb{N}$ and either position $\beta^{j}[i]$ is preceded or succeeded by $p^{m \cdot j / 2}=\beta^{j / 2}$. Therefore, (a) or (b) is satisfied.

Let $z_{0}=z$, let $z_{n}$ be the word $\tilde{z}$ after the $n$-th pumping step in the algorithm, and let $y=p^{m \cdot j-2}=\beta^{j} p^{-2}$. For each $n$, we will define a unique factorization

$$
z_{n}=x_{n, 0} y x_{n, 1} \cdots y x_{n, n}
$$

where $p$ is a suffix of $x_{n, i}$ for $i=0, \ldots, n-1$ and $p$ is a prefix of $x_{n, i}$ for $i=1, \ldots, n$. This factorization is defined inductively: naturally, we start with $x_{0,0}=z_{0}=z$. Assume $z_{n}$ is factorized in the above manner. Let $\alpha \beta \gamma=z_{n}[k ; k+\ell]$ be the factor, such that neither (a) nor (b) holds, which we replace in the $(n+1)$-st step (if there is no such factor, the algorithm terminates and we do not have to define $\left.z_{n+1}\right)$. By contradiction, assume that $\alpha$ starting at position $z_{n}[k]$ is covered by the $i$-th factor $y=p^{m \cdot j-2}$ in the factorization of $z_{n}$ for some $1 \leq i \leq n$. By the first observation, the factor $\beta \gamma=z_{n}[k+|\alpha| ; k+\ell]$ must overlap with $x_{i}$. However, as $p$ is a prefix of $x_{i}$, the factor $\beta=z_{n}[k+|\alpha| ; k+|\alpha \beta|]$ has to cover the prefix $p$ of $x_{i}$ or it has to cover one of the $p$ 's in $y$. This implies that $\gamma$ is preceded by $p^{m \cdot j / 2}=\beta^{j / 2}$ and (b) holds - contradiction. Symmetrically, $\gamma$ is not covered by one of the factors $y$ in $z_{n}$ neither.

Thus, $\beta=z_{n}[k+|\alpha| ; k+|\alpha \beta|]$ is covered by some $x_{n, i}$ in the factorization of $z_{n}$ and $x_{n, i}$ can be factorized $x_{n, i}=u \beta v$ where $u \neq \varepsilon$ and $v \neq \varepsilon$. Note that the length of $x_{n, i}$ has to be at least $|\beta|+2$. Now, let $x_{n+1, h}=x_{n, h}$ for $h=0, \ldots, i-1$, let $x_{n+1, h+1}=x_{n, h}$ for $h=i+2, \ldots, n$, let $x_{n+1, i}=u p$, and let $x_{n+1, i+1}=p v$. Observe that this defines the desired factorization. Also note that

$$
\left|x_{n+1, i}\right|=|u|+|p|=\left|x_{n, i}\right|-|\beta|-|v|+|p| \leq\left|x_{n, i}\right|-|v|<\left|x_{n, i}\right|
$$

and, symmetrically, $\left|x_{n+1, i+1}\right|<\left|x_{n, i}\right|$. Thus, in each pumping step, we replace one of the factors $x_{n, i}$ by two strictly shorter factors $x_{n+1, i}$ and $x_{n+1, i+1}$. As we have noted above, in a factor $x_{n, i}$ cannot be pumped anymore, if it is shorter than $|\beta|+2$. Eventually, all the the factors will be too short and the pumping algorithm will stop.

\section{Pixton's Variant of Splicing}

In this section we use the definition of the splicing operation as it was introduced in [17]. A triplet of words $r=\left(u_{1}, u_{2} ; v\right) \in\left(\Sigma^{*}\right)^{3}$ is called a (splicing) rule. The words $u_{1}$ and $u_{2}$ are called left and right site of $r$, respectively, and $v$ is the bridge of $r$. This splicing rule can be applied to two words $w_{1}=x_{1} u_{1} y_{1}$ and $w_{2}=x_{2} u_{2} y_{2}$, that each contain one of the sites, in order to create the new word $z=x_{1} v y_{2}$, see Figure 2. This operation is called splicing and it is denoted by $\left(w_{1}, w_{2}\right) \vdash_{r} z$.

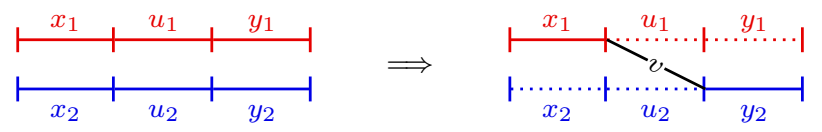

Figure 2: Splicing of the words $x_{1} u_{1} y_{1}$ and $x_{2} u_{2} y_{2}$ by the rule $r=\left(u_{1}, u_{2} ; v\right)$. 
For a rule $r$ we define the splicing operator $\sigma_{r}$ such that for a language $L$

$$
\sigma_{r}(L)=\left\{z \in \Sigma^{*} \mid \exists w_{1}, w_{2} \in L:\left(w_{1}, w_{2}\right) \vdash_{r} z\right\}
$$

and for a set of splicing rules $R$, we let

$$
\sigma_{R}(L)=\bigcup_{r \in R} \sigma_{r}(L)
$$

The reflexive and transitive closure of the splicing operator $\sigma_{R}^{*}$ is given by

$$
\sigma_{R}^{0}(L)=L, \quad \sigma_{R}^{i+1}(L)=\sigma_{R}^{i}(L) \cup \sigma_{R}\left(\sigma_{R}^{i}(L)\right), \quad \sigma_{R}^{*}(L)=\bigcup_{i \geq 0} \sigma_{R}^{i}(L) .
$$

A finite set of axioms $I \subseteq \Sigma^{*}$ and a finite set of splicing rules $R \subseteq\left(\Sigma^{*}\right)^{3}$ form a splicing system $(I, R)$. Every splicing system $(I, R)$ generates a language $L(I, R)=\sigma_{R}^{*}(I)$. Note that $L(I, R)$ is the smallest language which is closed under the splicing operator $\sigma_{R}$ and includes $I$. It is known that the language generated by a splicing system is regular, see [17]. A (regular) language $L$ is called a splicing language if a splicing system $(I, R)$ exists such that $L=L(I, R)$.

A rule $r$ is said to respect a language $L$ if $\sigma_{r}(L) \subseteq L$. It is easy to see that for any splicing system $(I, R)$, every rule $r \in R$ respects the generated language $L(I, R)$. Moreover, a rule $r \notin R$ respects $L(I, R)$ if and only if $L(I, R \cup\{r\})=L(I, R)$. We say a splicing $\left(w_{1}, w_{2}\right) \vdash_{r} z$ respects a language $L$ if $w_{1}, w_{2} \in L$ and $r$ respects $L$; obviously, this implies $z \in L$, too.

Pixton introduced this variant of splicing in order to give a simple proof for the regularity of languages generated by splicing systems. As Pixton's variant of splicing is more general than the classic splicing, defined in the introduction and in Section 4, his proof of regularity also applies to classic splicing systems. For a moment, let us call a classic splicing rule a quadruple and a Pixton splicing rule a triplet. Consider a quadruplet $r=\left(u_{1}, v_{1} ; u_{2}, v_{2}\right)$. It is easy to observe that whenever we can use $r$ in order to splice $w_{1}=x_{1} u_{1} v_{1} y_{1}$ with $w_{2}=x_{2} u_{2} v_{2} y_{2}$ to obtain the word $z=x_{1} u_{1} v_{2} y_{2}$, we can use the triplet $s=\left(u_{1} v_{1}, u_{2} v_{2} ; u_{1} v_{2}\right)$ in order to splice $\left(w_{1}, w_{2}\right) \vdash_{s} z$ as well. However, for a triplet $s=\left(u_{1}, u_{2} ; v\right)$ where $v$ is not a concatenation of a prefix of $u_{1}$ and a suffix of $u_{2}$, there is no quadruplet $r$ that can be used for the same splicings. Moreover, the class of classical splicing languages is strictly included in the class of Pixton splicing languages; e. g., the language

$$
L=c x^{*} a e+c x^{*} b e+d c x^{*} b e f
$$

over the alphabet $\{a, b, c, d, e, f, x\}$ is a Pixton splicing language but not a classical splicing language, see [4]. For the rest of this section we focus on Pixton's splicing variant and by a rule we always mean a triplet.

The main result of this section states that if a regular language $L$ is a splicing language, then it is created by a particular splicing system $(I, R)$ which only depends on the syntactic monoid of $L$.

Theorem 3.1. Let $L$ be a splicing language and $m=\left|M_{L}\right|$. The splicing system $(I, R)$ with $I=\Sigma^{<m^{2}+6 m} \cap L$ and

$$
R=\left\{r \in \Sigma^{<2 m} \times \Sigma^{<2 m} \times \Sigma^{<m^{2}+10 m} \mid r \text { respects } L\right\}
$$

generates the language $L=L(I, R)$.

As the language generated by the splicing system $(I, R)$ is constructible, Theorem 3.1 implies that the problem whether or not a given regular language is a splicing language is decidable. A detailed discussion of the decidability result is given in Section 5 .

Let $L$ be a formal language. Clearly, every set of words $J \subseteq L$ and set of rules $S$ where every rule in $S$ respects $L$ generates a subset $L(J, S) \subseteq L$. Therefore, in Theorem 3.1 the inclusion $L(I, R) \subseteq L$ is obvious. The rest of this section is devoted to the proof of the converse inclusion 
$L \subseteq L(I, R)$. Consider a splicing language $L$. One of the main techniques we use in the proof is that, whenever a word $z$ is created by a series of splicings from a set of words in $L$ and a set rules that respect $L$, then we can use a modified set of words from $L$ and modified rules which respect $L$ in order to obtain the same word $z$ by splicing. If $z$ is sufficiently long these words can be chosen such that they are all shorter than $z$ and the sites and bridges of the rules also satisfy certain length restrictions. Of course, our goal is to show that we can create $z$ by splicing from a subset of $I$ with rules which all satisfy the length bounds given by $R$ (as defined in Theorem 3.1). In Section 3.1 we will present techniques to obtain rules that respect $L$ from other rules respecting $L$ and we show how we can modify a single splicing step, such that the words used for splicing are not significantly longer than the splicing result. In Section 3.2 we use these techniques to modify series of splicings in the way described above (Lemma 3.8). Finally, in Section 3.3 we prove Theorem 3.1.

\subsection{Rule Modifications}

Let us start with the simple observation that we can extend the sites and the bridge of a rule $r$ such that the new rule respects all languages which are respected by $r$.

Lemma 3.2. Let $r=\left(u_{1}, u_{2} ; v\right)$ be a rule which respects a language $L$. For every word $x$, the rules $\left(x u_{1}, u_{2} ; x v\right),\left(u_{1} x, u_{2} ; v\right),\left(u_{1}, x u_{2} ; v\right)$, and $\left(u_{1}, u_{2} x ; v x\right)$ respect $L$ as well.

Proof. Let $s$ be any of the four rules $\left(x u_{1}, u_{2} ; x v\right),\left(u_{1} x, u_{2} ; v\right),\left(u_{1}, x u_{2} ; v\right)$, or $\left(u_{1}, u_{2} x ; v x\right)$. In order to prove that $s$ respects $L$ we have to show that, for all $w_{1}, w_{2} \in L$ and $z \in \Sigma^{*}$ such that $\left(w_{1}, w_{2}\right) \vdash_{s} z$, we have $z \in L$, too. Indeed, if $\left(w_{1}, w_{2}\right) \vdash_{s} z$, then $\left(w_{1}, w_{2}\right) \vdash_{r} z$ and as $r$ respects $L$, we conclude $z \in L$.

Henceforth, we will refer to the rules $\left(x u_{1}, u_{2} ; x v\right)$ and $\left(u_{1}, u_{2} x ; v x\right)$ as extensions of the bridge and to the rules $\left(u_{1} x, u_{2} ; v\right)$ and $\left(u_{1}, x u_{2} ; v\right)$ as extensions of the left and right site, respectively.

Next, for a language $L$, let us investigate the syntactic class of a rule $r=\left(u_{1}, u_{2} ; v\right)$. The syntactic class (with respect to $L$ ) of $r$ is the set of rules $[r]_{L}=\left[u_{1}\right]_{L} \times\left[u_{2}\right]_{L} \times[v]_{L}$ and two rules $r$ and $s$ are syntactically congruent (with respect to $L$ ), denoted by $r \sim_{L} s$, if $s \in[r]_{L}$.

Lemma 3.3. Let $r$ be a rule which respects a language $L$. Every rule $s \in[r]_{L}$ respects $L$.

Proof. Let $r=\left(u_{1}, u_{2} ; v\right)$ and $s=\left(\tilde{u}_{1}, \tilde{u}_{2} ; \tilde{v}\right)$. Thus, $u_{i} \sim_{L} \tilde{u}_{i}$ for $i=1,2$ and $v \sim_{L} \tilde{v}$. For $\tilde{w}_{1}=x_{1} \tilde{u}_{1} y_{1} \in L$ and $\tilde{w}_{2}=x_{2} \tilde{u}_{2} y_{2} \in L$ we have to show that $\tilde{z}=x_{1} \tilde{v} y_{2} \in L$. For $i=1,2$, let $w_{i}=x_{i} u_{i} y_{i}$ and note that $w_{i} \sim_{L} \tilde{w}_{i}$; hence, $w_{i} \in L$. Furthermore, $\left(w_{1}, w_{2}\right) \vdash_{r} x_{1} v y_{2}=z \in L$ as $r$ respects $L$ and $\tilde{z} \in L$ as $z \sim_{L} \tilde{z}$.

Consider a splicing $\left(x_{1} u_{1} y_{1}, x_{2} u_{2} y_{2}\right) \vdash_{r} x_{1} v y_{2}$ which respects a regular language $L$ as shown in Figure 3 left side. The factors $u_{1} y_{1}$ and $x_{2} u_{2}$ may be relatively long but they do not occur as factors in the resulting word $x_{1} v y_{2}$. In particular, it is possible that two long words are spliced and the outcome is a relatively short word. Using Lemmas 3.2 and 3.3, we can find shorter words in $L$ and a modified splicing rule which can be used to obtain $x_{1} v y_{2}$.

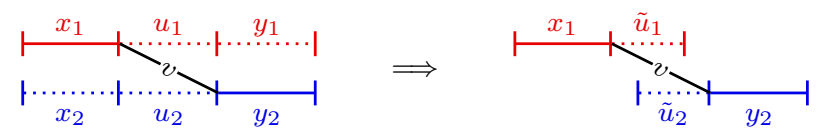

Figure 3: The factors $u_{1} y_{1}$ and $x_{2} u_{2}$ can be replaced by short words.

Lemma 3.4. Let $r=\left(u_{1}, u_{2} ; v\right)$ be a rule which respects a regular language $L$ and $w_{1}=x_{1} u_{1} y_{1} \in$ $L, w_{2}=x_{2} u_{2} y_{2} \in L$. There is a rule $s=\left(\tilde{u}_{1}, \tilde{u}_{2} ; v\right)$ which respects $L$ and words $\tilde{w}_{1}=x_{1} \tilde{u}_{1} \in L$, $\tilde{w}_{2}=\tilde{u}_{2} y_{2} \in L$ such that $\left|\tilde{u}_{1}\right|,\left|\tilde{u}_{2}\right|<\left|M_{L}\right|$. More precisely, $\tilde{u}_{1} \in\left[u_{1} y_{1}\right]_{L}$ and $\tilde{u}_{2} \in\left[x_{2} u_{2}\right]_{L}$.

In particular, whenever $\left(w_{1}, w_{2}\right) \vdash_{r} x_{1} v y_{2}=z$, there is a splicing $\left(\tilde{w}_{1}, \tilde{w}_{2}\right) \vdash_{s} z$ which respects $L$ where $\tilde{w}_{1}, \tilde{w}_{2}$, and s have the properties described above. 
Proof. By Lemma 3.2, the rule $\left(u_{1} y_{1}, x_{2} u_{2} ; v\right)$ respects $L$. Choose $\tilde{u}_{1} \in\left[u_{1} y_{1}\right]_{L}$ and $\tilde{u}_{2} \in\left[x_{2} u_{2}\right]_{L}$ as shortest words from the syntactic classes, respectively; as such, $\left|\tilde{u}_{1}\right|,\left|\tilde{u}_{2}\right|<\left|M_{L}\right|$ (Lemma 2.2) and $\tilde{w}_{1}=x_{1} \tilde{u}_{1} \in L, w_{2}=\tilde{u}_{2} y_{2} \in L$. Furthermore, by Lemma 3.3, $s=\left(\tilde{u}_{1}, \tilde{u}_{2} ; v\right)$ respects $L$.

Another way of modifying a splicing $\left(w_{1}, w_{2}\right) \vdash_{r} z$ is to extend the bridge of $r$ to the left until it covers a prefix of $w_{1}$. Afterwards, we can use the same method we used in Lemma 3.4 and replace $w_{1}$ by a short word, see Figure 4. As the splicing operation is symmetric, we can also extend the bridge of $r$ rightwards and replace $w_{2}$ by a short word, even though Lemma 3.5 does not explicitly state this.

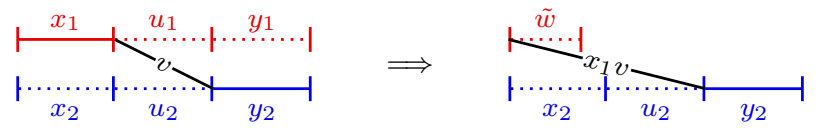

Figure 4: The word $x_{1} u_{1} y_{1}$ can be replaced by a short word as long as we extend the bridge of the splicing rule accordingly.

Lemma 3.5. Let $r=\left(u_{1}, u_{2} ; v\right)$ be a rule which respects a regular language $L$ and let $w_{1}=$ $x_{1} u_{1} y_{1} \in L$. Every rule $s=\left(\tilde{w}, u_{2} ; x_{1} v\right)$, where $\tilde{w} \in\left[w_{1}\right]_{L} \subseteq L$, respects $L$. In particular, there is a rule $s$, as above, where $|\tilde{w}|<\left|M_{L}\right|$.

Proof. By Lemma 3.2, we see that $\left(x_{1} u_{1} y_{1}, u_{2} ; x_{1} v\right)$ respects $L$ and, by Lemma $3.3, s=\left(\tilde{w}, u_{2} ; x_{1} v\right)$ respects $L$. If $\tilde{w} \in\left[w_{1}\right]_{L}$ is a shortest word from the set, then $|\tilde{w}|<\left|M_{L}\right|$ by Lemma 2.2.

\subsection{Series of Splicings}

We are now investigating words which are created by a series of successive splicings which all respect a regular language $L$. Observe, that if a word is created by two (or more) successive splicings, but the bridges of the rules do not overlap in the generated word, then the order of these splicings is irrelevant. The notation in Remark 3.6 is the same as in the Figure 5.

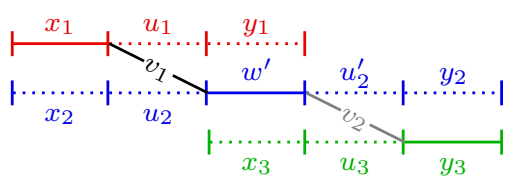

Figure 5: The word $x_{1} v_{1} w^{\prime} v_{2} y_{3}$ can be created either by using the right splicing first or by using the left splicing first.

Remark 3.6. Consider rules $r=\left(u_{1}, u_{2} ; v_{1}\right)$ and $s=\left(u_{2}^{\prime}, u_{3} ; v_{2}\right)$ and words $w_{1}=x_{1} u_{1} y_{1}, w_{2}=$ $x_{2} u_{2} w^{\prime} u_{2}^{\prime} y_{2}$, and $w_{3}=x_{3} u_{3} y_{3}$. The word $z=x_{1} v_{1} w^{\prime} v_{2} y_{3}$ can be obtained by the splicings

$$
\begin{array}{lll}
\left(w_{1}, w_{2}\right) \vdash_{r} x_{1} v_{1} w^{\prime} u_{2}^{\prime} y_{2}=z^{\prime}, & \left(z^{\prime}, w_{3}\right) \vdash_{s} z \quad \text { as well as } \\
\left(w_{2}, w_{3}\right) \vdash_{s} x_{2} u_{2} w^{\prime} v_{2} y_{3}=z^{\prime \prime}, & \left(w_{1}, z^{\prime \prime}\right) \vdash_{r} z, &
\end{array}
$$

which makes the order of the splicing steps irrelevant.

Now, consider a word $z$ which is created by two successive splicings from words $w_{i}=x_{i} u_{i} y_{i}$ for $i=1,2,3$ as in Figure 6. If no factor of $w_{1}$ or of the bridge in the first splicing is a part of $z$, then we can find another splicing rule $s$ such that $\left(w_{3}, w_{2}\right) \vdash_{s} z$ and the bridge of $s$ is the bridge used in the second splicing.

Lemma 3.7. Let $L$ be a language, $w_{i}=x_{i} u_{i} y_{i} \in L$ for $i=1,2,3$, and $r_{1}=\left(u_{1}, u_{2} ; v_{1}\right), r_{2}=$ $\left(u_{3}, u_{4} ; v_{2}\right)$ be rules respecting $L$. If there are splicings

$$
\left(w_{1}, w_{2}\right) \vdash_{r_{1}} x_{1} v_{1} y_{2}=w_{4}=x_{4} u_{4} y_{4}, \quad\left(w_{3}, w_{4}\right) \vdash_{r_{2}} x_{3} v_{2} y_{4}=z
$$

where $y_{4}$ is a suffix of $y_{2}$, then there is a rule $s=\left(u_{3}, \tilde{u}_{2} ; v_{2}\right)$ which respects $L$ and $\left(w_{3}, w_{2}\right) \vdash_{s} z$. 


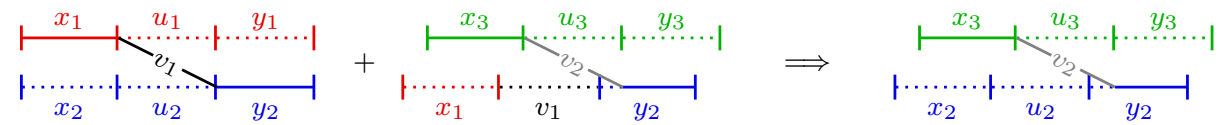

Figure 6: Two successive splicings can be replaced by one splicing in the case when the factor $x_{1}$ and the bridge $v_{1}$ do not contribute to the resulting word.

Proof. By extending the bridge $v_{1}$ of $r_{1}$ and the right site $u_{4}$ of $r_{2}$ (Lemma 3.2), we may assume the factors $v_{1}$ and $u_{4}$ match in $w_{4}$ : let $w_{4}[i, j]=v_{1}$ and $w_{4}\left[i^{\prime}, j^{\prime}\right]=u_{4}$,

- if $i<i^{\prime}$ we extend $u_{4}$ in $r_{2}$ to the left by $i^{\prime}-i$ letters,

- if $i>i^{\prime}$ we extend $v_{1}$ in $r_{1}$ to the left by $i-i^{\prime}$ letters and we extend $u_{1}$ accordingly, and

- we extend $v_{1}$ in $r_{1}$ to the right by $j^{\prime}-j$ letters and we extend $u_{2}$ accordingly; Note that $j^{\prime} \geq j$ as $y_{4}$ is a suffix of $y_{2}$.

Clearly, the extended factors $v_{1}$ and $u_{4}$ match in $w_{4}$. The left site $u_{3}$ and the bridge $v_{2}$ of $r_{2}$ are not modified by this extension. Additionally, we have $x_{1}=x_{4}$ and $y_{2}=y_{4}$. Let $s=\left(u_{3}, u_{2} ; v_{2}\right)$ (where $u_{2}$ is the extended right site of $r_{1}$ ). As desired, $\left(w_{3}, w_{2}\right) \vdash_{s} x_{3} v_{2} y_{4}=z$ since $w_{2}=x_{2} u_{2} y_{4}$.

Next, let us prove that $s$ respects $L$. Let $w_{i}^{\prime}=x_{i}^{\prime} u_{i} y_{i}^{\prime} \in L$ for $i=2,3$. If for all those words $x_{3}^{\prime} v_{2} y_{2}^{\prime} \in L$, then $s$ respects $L$. Indeed, we may splice

$$
\left(w_{1}, w_{2}^{\prime}\right) \vdash_{r_{1}} x_{1} v_{1} y_{2}^{\prime}=x_{1} u_{4} y_{2}^{\prime}, \quad\left(w_{3}^{\prime}, x_{1} u_{4} y_{2}^{\prime}\right) \vdash_{r_{2}} x_{3}^{\prime} v_{2} y_{2}^{\prime} .
$$

Therefore, $x_{3}^{\prime} v_{2} y_{2}^{\prime} \in L$ and $s$ respects $L$.

Consider a splicing system $(J, S)$ and its generated language $L=L(J, S)$. Let $n$ be the length of the longest word in $J$ and let $\mu$ be the length-lexicographic largest word that is a component of a rule in $S$. Define $W_{\mu}=\left\{w \in \Sigma^{*} \mid w \leq_{\ell \ell} \mu\right\}$ as the set of all words that are at most as large as $\mu$, in length-lexicographical order. Furthermore, let $I=\Sigma^{\leq n} \cap L$ be a set of axioms and let

$$
R=\left\{r \in W_{\mu}^{3} \mid r \text { respects } L\right\}
$$

be a set of rules. It is not difficult to see that $J \subseteq I, S \subseteq R$, and $L=L(I, R)$. Whenever convenient, we may assume that a splicing language $L$ is generated by a splicing system which is of the form of $(I, R)$.

Now, consider the creation of a word $x z y \in L$ by splicing in $(I, R)$. The creation of $x z y$ can be traced back to a word $z_{1}=x_{1} z y_{1}$ where either $z_{1} \in I$ or where $z_{1}$ is created by a splicing that affects $z$, i.e., the bridge in this splicing overlaps with the factor $z$ in $x_{1} z y_{1}$. The next lemma describes this creation of $x z y=z_{k+1}$ by $k$ splicings in $(I, R)$, and shows that we can choose the rules and words which are used to create $z_{k+1}$ from $z_{1}$ such that the words and bridges of rules are not significantly longer than $\ell=\max \{|x|,|y|\}$.

Lemma 3.8. Let $L$ be a splicing language, let $\ell, n \in \mathbb{N}$, let $m=\left|M_{L}\right|$, and let $\mu$ be a word with $|\mu| \geq \ell+2 m$ such that for $I=\Sigma^{\leq n} \cap L$ and $R=\left\{r \in W_{\mu}^{3} \mid r\right.$ respects $\left.L\right\}$ we have $L=L(I, R)$.

Let $z_{k+1}=x_{k+1} z y_{k+1}$, with $\left|x_{k+1}\right|,\left|y_{k+1}\right| \leq \ell$, be a word that is created by $k$ splicings from a word $z_{1}=x_{1} z y_{1}$ where either $z_{1} \in I$ or $z_{1}$ is created by a splicing $\left(w_{0}, w_{0}^{\prime}\right) \vdash_{s} z_{1}$ with $w_{0}, w_{0}^{\prime} \in L$, $s \in R$, and the bridge of $s$ overlaps with $z$ in $z_{1}$. Furthermore, for $i=1, \ldots, k$ the intermediate splicings are either

(i) $\left(w_{i}, z_{i}\right) \vdash_{r_{i}} x_{i+1} z y_{i+1}=z_{i+1}, w_{i} \in L, r_{i} \in R, y_{i+1}=y_{i}$, and the bridge of $r_{i}$ is covered by the prefix $x_{i+1}$ or

(ii) $\left(z_{i}, w_{i}\right) \vdash_{r_{i}} x_{i+1} z y_{i+1}=z_{i+1}, w_{i} \in L, r_{i} \in R, x_{i+1}=x_{i}$, and the bridge of $r_{i}$ is covered by the suffix $y_{i+1}$. 
There are rules and words creating $z_{k+1}$, as above, satisfying in addition:

1. There is $k^{\prime} \leq k$ such that for $i=1, \ldots, k^{\prime}$ all splicings are of the form (i) and for $i=$ $k^{\prime}+1, \ldots, k$ all splicings are of the form (ii).

2. For $i=1, \ldots, k$ the following bounds apply: $\left|x_{i}\right|,\left|y_{i}\right|<\ell+2 m,\left|w_{i}\right|<m, r_{i} \in \Sigma^{<2 m} \times$ $\Sigma^{<2 m} \times \Sigma^{<\ell+m}$.

In particular, if $n \geq m$, then $w_{1}, \ldots, w_{k} \in I$.

Proof. Statement 1 follows by Remark 3.6 Note that if $k=0$, then statement 2 is trivially true. By the first statement, $x_{k^{\prime}+1}=x_{k^{\prime}+2}=\cdots=x_{k+1}$ and $y_{1}=y_{2}=\cdots=y_{k^{\prime}+1}$. Let us consider the splicings of the form (i) which are the steps $i=1, \ldots, k^{\prime}$. The notations we employ in order to prove the second statement for $i=1, \ldots, k^{\prime}$ are chosen to match the notations in Figure 7 .

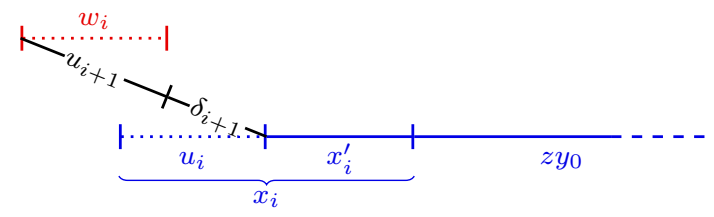

Figure 7: The $i$-th splicing step in the proof of Lemma 3.8 where $v_{i}=u_{i+1} \delta_{i+1}$ and $x_{i+1}=$ $u_{i+1} \delta_{i+1} x_{i}^{\prime}$.

Let $r_{i}=\left(w_{i}, u_{i} ; v_{i}\right)$ where $w_{i} \in \Sigma^{<m} \cap L$ (Lemma 3.5) and $x_{i}=u_{i} x_{i}^{\prime}$; (by Lemma 3.2, we extended the site $u_{i}$ to cover a prefix of $x_{i}$ ) such that $u_{i+1} x_{i+1}^{\prime}=v_{i} x_{i}^{\prime}$ with $u_{k^{\prime}+1}=\varepsilon$ and $x_{k^{\prime}+1}^{\prime}=x_{k^{\prime}+1}=x_{k+1}$. Lemma 3.7 justifies the assumption that every splicing occurs at the left of the preceding splicing, i. e., $x_{i}^{\prime}$ is a proper suffix of $x_{i+1}^{\prime}$. Note that, as $\left|x_{k^{\prime}+1}^{\prime}\right| \leq \ell$, the length of $x_{i}^{\prime}$ is bounded by $\ell$. Now, choose $\delta_{i+1}$ such that $x_{i+1}^{\prime}=\delta_{i+1} x_{i}^{\prime}$; thus, $u_{i+1} \delta_{i+1}=v_{i}$.

For $i=2, \ldots, k^{\prime}$ we replace $u_{i}$ by a shortest word from $\left[u_{i}\right]_{L}$. Note that this does not change the fact that all rules respect $L$ (Lemma 3.3). We also replace the prefix of $x_{i}$ and $v_{i-1}$ by this factor. (There is no need to change $v_{k^{\prime}}$ as $\left|v_{k^{\prime}}\right|=\left|\delta_{k^{\prime}+1}\right| \leq\left|x_{k+1}\right| \leq \ell$.) Therefore, $\left|x_{i}\right|<\left|x_{i}^{\prime}\right|+m<\ell+m$ and $r_{i} \in \Sigma^{<m} \times \Sigma^{<m} \times \Sigma^{<\ell+m}$ if $i \neq 1$ (Lemma 2.2). We do not change $u_{1}$ yet as this may affect the splicing $\left(w_{0}, w_{0}^{\prime}\right) \vdash_{s} z_{1}$ if it exists. Note that, for $i=2, \ldots, k^{\prime}$, we have actually proven a stronger bound than claimed in statement 2 of Lemma 3.8. Even though we have not proven the bound for $r_{1}$ yet, we have already established $r_{1} \in \Sigma^{<m} \times \Sigma^{*} \times \Sigma^{<\ell+m}$. Symmetrically, we can consider statement 2 to be proven for $i=k^{\prime}+2, \ldots, k$, i. e., only the prefix $x_{1}$ and the suffix $y_{1}=y_{k^{\prime}+1}$ have not been modified yet.

Now, let $x_{1}=u_{1} x_{1}^{\prime}$ (as above) and, symmetrically, let $y_{1}=y_{k^{\prime}+1}^{\prime} u_{k^{\prime}+1}$ where $u_{k^{\prime}+1}$ is the left site of $r_{k^{\prime}+1}$. If $k^{\prime}=0$ (or $k^{\prime}=k$ ), then $u_{1}$ (resp. $u_{k^{\prime}+1}$ ) can be considered empty and $x_{1}^{\prime}=x_{k+1}\left(\right.$ resp. $\left.y_{k^{\prime}+1}^{\prime}=y_{k+1}\right)$. If $z_{1} \in I$ we replace $u_{1}$ and $u_{k^{\prime}+1}$ by shortest words from their syntactic classes, respectively, and the claim holds. Otherwise, $\left(w_{0}, w_{0}^{\prime}\right) \vdash_{s} z_{1}$ where $s=\left(u_{0}, u_{0}^{\prime}, v\right)$, $w_{0}=x u_{0}$, and $w_{0}^{\prime}=u_{0}^{\prime} y$, by Lemma 3.4. Thus,

$$
z_{1}=u_{1} x_{1}^{\prime} z y_{k^{\prime}+1}^{\prime} u_{k^{\prime}+1}=x v y .
$$

In the case when $v$ does not overlap with the prefix $u_{1}$ of $z_{1}$, replace $u_{1}$ by a shortest word from its syntactic class. If $v$ and the prefix $u_{1}$ overlap, let $u_{1}=\delta_{1} \delta_{2}$ such that $\delta_{2}$ is the overlap and replace $\delta_{1}$ and $\delta_{2}$ by a shortest word from their syntactic classes, respectively. In both cases, $\left|u_{1}\right|<2 m$ (Lemma 2.2) and if $v$ was modified, it got shorter; hence, we still have $v \in W_{\mu}$. Observe that $\left|x_{1}\right|<\ell+2 m$ and $r_{1} \in \Sigma^{<m} \times \Sigma^{<2 m} \times \Sigma^{<\ell+m}$. Analogously, $u_{k^{\prime}+1}$ and $r_{k^{\prime}+1}$ can be treated in order to conclude the prove of statement 2 .

\subsection{Proof of Theorem 3.1}

Let $L$ be a splicing language and $m=\left|M_{L}\right|$. Throughout this section, by $\sim$ we denote the equivalence relation $\sim_{L}$ and by $[\cdot]$ we denote the corresponding equivalence classes $[\cdot]_{L}$. 
Recall that Theorem 3.1 claims that the splicing system $(I, R)$ with $I=\Sigma^{<m^{2}+6 m} \cap L$ and

$$
R=\left\{r \in \Sigma^{<2 m} \times \Sigma^{<2 m} \times \Sigma^{<m^{2}+10 m} \mid r \text { respects } L\right\}
$$

generates $L$. The proof is divided in two parts. In the first part, Lemma 3.9, we prove that $L$ is generated by a splicing system $\left(I, R^{\prime}\right)$ where all sites of rules in $R^{\prime}$ are shorter than $2 m$, but we do not care about the lengths of the bridges. The second part will then conclude the proof by showing that there are no rules in $R^{\prime}$ with bridges of length greater than or equal to $m^{2}+10 m$ which are essential for the creation of the language $L$ by splicing.

Lemma 3.9. Let $L, m$, and $I$ as above. There is $n \in \mathbb{N}$ such that the splicing system $\left(I, R^{\prime}\right)$ with

$$
R^{\prime}=\left\{r \in \Sigma^{<2 m} \times \Sigma^{<2 m} \times \Sigma^{\leq n} \mid r \text { respects } L\right\}
$$

generates the language $L=L\left(I, R^{\prime}\right)$.

Proof. As $I \subseteq L$ and every rule in $R^{\prime}$ respects $L$, it is clear that $L\left(I, R^{\prime}\right) \subseteq L$ for any $n$; we only need to prove the converse inclusion.

As $L$ is a splicing language, $L=L(J, S)$ for some splicing system $(J, S)$. Let $n$ be larger than the length of every bridge of every rule in $S$ and $n \geq 4 m^{2}$.

In order to prove $L \subseteq L\left(I, R^{\prime}\right)$ we use induction on the length of words in $L$. For all $w \in L$ with $|w|<m^{2}+6 m$, by definition, $w \in I \subseteq L\left(I, R^{\prime}\right)$.

Now, consider $w \in L$ with $|w| \geq m^{2}+6 m$. The induction hypothesis states that every word $w^{\prime} \in L$ with $\left|w^{\prime}\right|<|w|$ belongs to $L\left(I, R^{\prime}\right)$. Factorize $w=x \alpha \beta \gamma \delta y$ such that $|x|,|y|=3 m$, $|\alpha \beta \gamma|=m^{2},|\beta| \geq 1, \alpha \sim \alpha \beta$, and $\gamma \sim \beta \gamma$.

The proof idea is to use a pumping argument on $\alpha \beta \gamma$ in order to obtain a very long word. This word has to be created by a series of splicings in $(J, S)$. We show that these splicings can be modified in order to create $w$ by splicing from a set of strictly shorter words and with rules from $R^{\prime}$. Then, the induction hypothesis implies $w \in L\left(I, R^{\prime}\right)$.

Choose $j$ sufficiently large $(j>n$ and $J$ does not contain words of length $j$ or more). We let $z=\alpha \beta^{j} \gamma \delta$ and investigate the creation of $x z y \in L$. As $z$ is not a factor of a words in $J$, every word in $L$ which contains $z$ is created by some splicing in $(J, S)$. Thus, we can trace back the creation of $x z y$ by splicing to the point where the factor $z$ is affected for the last time. Let $z_{k+1}=x_{k+1} z y_{k+1}$, where $x_{k+1}=x$ and $y_{k+1}=y$, be created by $k$ splicings from a word $z_{1}=x_{1} z y_{1}$ where $x_{1} z y_{1}$ is created by a splicing $\left(w_{0}, w_{0}^{\prime}\right) \vdash_{s} z_{1}$ with $w_{0}, w_{0}^{\prime} \in L, s \in S$, and the bridge of $s$ overlaps with $z$ in $z_{1}$. Furthermore, for $i=1, \ldots, k$ the intermediate splicings are either

(i) $\left(w_{i}, z_{i}\right) \vdash_{r_{i}} x_{i+1} z y_{i+1}=z_{i+1}, w_{i} \in L, r_{i} \in S, y_{i+1}=y_{i}$, and the bridge of $r_{i}$ is covered by the prefix $x_{i+1}$ or

(ii) $\left(z_{i}, w_{i}\right) \vdash_{r_{i}} x_{i+1} z y_{i+1}=z_{i+1}, w_{i} \in L, r_{i} \in S, x_{i+1}=x_{i}$, and the bridge of $r_{i}$ is covered by the suffix $y_{i+1}$.

Following Lemma 3.8 (with $\ell=3 m$ ), we may assume that $w_{1}, \ldots, w_{k} \in I, r_{1}, \ldots, r_{k} \in \Sigma^{<2 m} \times$ $\Sigma^{<2 m} \times \Sigma^{<4 m}$, thus $r_{1}, \ldots, r_{k} \in R^{\prime}$, and $\left|x_{1}\right|,\left|y_{1}\right|<5 m$. Furthermore, we may use the same words and rules in order to create $w=x_{k+1} \alpha \beta \gamma \delta y_{k+1}$ from $x_{1} \alpha \beta \gamma \delta y_{1}$ by splicing, i. e., if $x_{1} \alpha \beta \gamma \delta y_{1}$ belongs to $L\left(I, R^{\prime}\right)$, so does $w$.

Now, consider the first splicing $\left(w_{0}, w_{0}^{\prime}\right) \vdash_{s} z_{1}=x_{1} z y_{1}$. By Lemma 3.4, we assume $s=$ $\left(u_{1}, u_{2} ; v\right)$ such that $w_{0}=x u_{1}, w_{0}^{\prime}=u_{2} y$ and $\left|u_{1}\right|,\left|u_{2}\right|<m$ ( $x$ and $y$ are newly chosen words). Hence,

$$
z_{0}=x v y=x_{1} \alpha \beta^{j} \gamma \delta y_{1} .
$$

where $x$ is a proper prefix of $x_{1} \alpha \beta^{j} \gamma \delta$ and $y$ is a proper suffix of $\alpha \beta^{j} \gamma \delta y_{1}$.

We will now pump down the factor $\beta^{j}$ to $\beta$ in order to obtain the words $\tilde{x}, \tilde{v}, \tilde{y}$ from $x, v, y$, respectively, as follows:

1. If $v$ overlaps with $\beta^{j}$ but does neither cover $\alpha$ nor $\gamma$, extend $v$ (Lemma 3.2) such that $v=\alpha \beta^{j} \gamma$. Observe that, now, the factor $\alpha \beta^{j} \gamma$ is covered by either $x v$ or $v y$. 
2. If $\alpha \beta^{j}$ or $\beta^{j} \gamma$ is covered by one of $x, v$, or $y$, then replace this factor by $\alpha \beta$ or $\beta \gamma$, respectively. Otherwise, by symmetry, assume that $\alpha \beta^{j} \gamma$ is covered by $x v$ and, therefore, we can factorize

$$
x=x_{1} \alpha \beta^{j_{1}} \beta_{1} \quad v=\beta_{2} \beta^{j_{2}} \gamma v^{\prime}
$$

where $\beta_{1} \beta_{2}=\beta$ and $j_{1}+j_{2}+1=j$. The results of pumping are the words $\tilde{x}=x_{1} \alpha \beta_{1}, \tilde{v}=\beta_{2} \gamma v^{\prime}$, and $\tilde{y}=y$.

Let $\tilde{u}_{1}$ and $\tilde{u}_{2}$ be the sites of $s$ that may have been altered due to the extension of $v$ and, by Lemma 3.4, assume $\left|\tilde{u}_{1}\right|,\left|\tilde{u}_{2}\right|<m$. If we used an extension for $v$, then $|\tilde{v}|=m^{2}$. No matter whether we used an extension, $t=\left(\tilde{u}_{1}, \tilde{u}_{2} ; \tilde{v}\right) \in R^{\prime}$ and $\left(\tilde{x} \tilde{u}_{1}, \tilde{u}_{2} \tilde{y}\right) \vdash_{t} x_{1} \alpha \beta \gamma \delta y_{1}$ as desired. Observe that $\tilde{x}$ is a prefix of $x_{1} \alpha \beta \gamma \delta$ and $\tilde{y}$ is a suffix of $\alpha \beta \gamma \delta y_{1}$ and recall that $\left|x_{1}\right|,\left|y_{1}\right|<5 m$. Therefore, $\left|\tilde{x} \tilde{u}_{1}\right|,\left|\tilde{u}_{2} \tilde{y}\right|<|\alpha \beta \gamma \delta|+6 m=|w|$ and, by induction hypothesis, $\tilde{x} \tilde{u}_{1}$ and $\tilde{u}_{2} \tilde{y}$ belong to $L\left(I, R^{\prime}\right)$. We conclude that $x_{1} \alpha \beta \gamma \delta y_{1}$ as well as $w$ belong to $L\left(I, R^{\prime}\right)$.

We are now prepared to prove the main result.

Proof of Theorem 3.1. Recall that for a splicing language $L$ with $m=\left|M_{L}\right|$, we intend to prove that the splicing system $(I, R)$ with $I=\Sigma^{<m^{2}+6 m} \cap L$ and

$$
R=\left\{r \in \Sigma^{<2 m} \times \Sigma^{<2 m} \times \Sigma^{<m^{2}+10 m} \mid r \text { respects } L\right\}
$$

generates the language $L=L(I, R)$. Obviously, $L(I, R) \subseteq L$. By Lemma 3.9, there is a finite set of rules $R^{\prime} \subseteq \Sigma^{<2 m} \times \Sigma^{<2 m} \times \Sigma^{*}$ such that $L\left(I, R^{\prime}\right)=L$.

For a word $\mu$ we let $W_{\mu}=\left\{w \in \Sigma^{*} \mid w \leq_{\ell \ell} \mu\right\}$, as we did before. Define the set of rules where every component is length-lexicographically bounded by $\mu$

$$
R_{\mu}=\left\{r \in \Sigma^{<2 m} \times \Sigma^{<2 m} \times W_{\mu} \mid r \text { respects } L\right\}
$$

and the language $L_{\mu}=L\left(I, R_{\mu}\right)$; clearly, $L_{\mu} \subseteq L$. For two words $\mu \leq_{\ell \ell} v$ we see that $R_{\mu} \subseteq R_{v}$, and hence, $L_{\mu} \subseteq L_{v}$. Thus, if $L_{\mu}=L$ for some word $\mu$, then for all words $v$ with $\mu \leq_{\ell \ell} v$, we have $L_{v}=L$. As $L=L\left(I, R^{\prime}\right)$, there exists a word $\mu$ such that $L_{\mu}=L$. Let $\mu$ be the smallest word, in the length-lexicographic order, such that $L_{\mu}=L$. Note that if $|\mu|<m^{2}+10$, then $R_{\mu} \subseteq R$ and $L=L_{\mu} \subseteq L(I, R)$. For the sake of contradiction assume $|\mu| \geq m^{2}+10 m$. Let $\nu$ be the next-smaller word than $\mu$, in the length-lexicographic order, and let $S=R_{\nu}$. Note that $L(I, S) \subsetneq L$ and $R_{\mu} \backslash S$ contains only rules whose bridges are $\mu$.

Choose $w$ from $L \backslash L(I, S)$ as a shortest word, i. e., for all $w^{\prime} \in L$ with $\left|w^{\prime}\right|<|w|$, we have $w^{\prime} \in L(I, S)$. Factorize $w=x z y$ with $|x|=|y|=3 m$; note that $|w| \geq m^{2}+6 m$ since, otherwise, $w \in I$. Factorize $\mu=\delta_{1} \alpha \beta \gamma \delta_{2}$ with $\left|\delta_{1}\right|,\left|\delta_{2}\right| \geq 5 m,|\alpha \beta \gamma|=m^{2}, \beta \neq \varepsilon, \alpha \sim \alpha \beta$, and $\gamma \sim \beta \gamma$, by Lemma 2.1.

Next, we will use a pumping argument on all factors $\alpha \beta \gamma$ in $z$. As in the proof of Lemma 3.9, this new word has to be created by a series of splicings in $\left(I, R_{\mu}\right)$ and we will show that these splicings can be modified in order to create $w$ from strictly shorter words and with rules from $S$. This will contradict the assumption that $w$ is a shortest word from $L \backslash L(I, S)$.

Let $j$ be a sufficiently large even number $(j>4|\mu|+|z|$ will do). We define a word $\tilde{z}$ which is the result of applying the pumping algorithm from Lemma 2.3 on $z$, as discussed in Section 2.1. The pumping algorithm replaces the occurrences of $\alpha \beta \gamma$ in $z$ by $\alpha \beta^{j} \gamma$ such that for every factor $\tilde{z}\left[k, k+m^{2}\right]=\alpha \beta \gamma$, either

(a) $\alpha \beta^{j / 2}$ is a factor of $\tilde{z}$ starting at position $\tilde{z}[k]$ or

(b) $\beta^{j / 2} \gamma$ is a factor of $\tilde{z}$ ending at position $\tilde{z}\left[k+m^{2}\right]$

holds. In particular, if $\delta_{1} \alpha \beta \gamma \delta_{2}$ is a factor of $\tilde{z}$ either (a) $\gamma \delta_{2}$ is a prefix of a word in $\beta^{+}$or (b) $\delta_{1} \alpha$ is a suffix of a word in $\beta^{+}$. By induction and as $\alpha \beta \gamma \sim \alpha \beta^{j} \gamma$, it is easy to see that $z \sim \tilde{z}$ and $x \tilde{z} y \in L$.

Let us trace back the creation of $x \tilde{z} y \in L$ by splicing in $\left(I, R_{\mu}\right)$ to a word $x_{1} \tilde{z} y_{1}$ where either $x_{1} \tilde{z} y_{1} \in I$ or where $x_{1} \tilde{z} y_{1}$ is created by a splicing that affects $\tilde{z}$. Let $z_{k+1}=x_{k+1} \tilde{z} y_{k+1}$, where 
$x_{k+1}=x$ and $y_{k+1}=y$, be created by $k$ splicings from a word $z_{1}=x_{1} \tilde{z} y_{1}$ where either $x_{1} \tilde{z} y_{1} \in I$ or $x_{1} \tilde{z} y_{1}$ is created by a splicing $\left(w_{0}, w_{0}^{\prime}\right) \vdash_{s} z_{1}$ with $w_{0}, w_{0}^{\prime} \in L, s \in R_{\mu}$, and the bridge of $s$ overlaps with $\tilde{z}$. Furthermore, for $i=1, \ldots, k$ the intermediate splicings are either

(i) $\left(w_{i}, z_{i}\right) \vdash_{r_{i}} x_{i+1} \tilde{z} y_{i+1}=z_{i+1}, w_{i} \in L, r_{i} \in R_{\mu}, y_{i+1}=y_{i}$, and the bridge of $r_{i}$ is covered by the prefix $x_{i+1}$ or

(ii) $\left(z_{i}, w_{i}\right) \vdash_{r_{i}} x_{i+1} \tilde{z} y_{i+1}=z_{i+1}, w_{i} \in L, r_{i} \in R_{\mu}, x_{i+1}=x_{i}$, and the bridge of $r_{i}$ is covered by the suffix $y_{i+1}$.

Following Lemma 3.8 (with $\ell=3 m$ ), we may assume that $w_{1}, \ldots, w_{k} \in I, r_{1}, \ldots, r_{k} \in \Sigma^{<2 m} \times$ $\Sigma^{<2 m} \times \Sigma^{<4 m}$, thus $r_{1}, \ldots, r_{k} \in S$, and $\left|x_{1}\right|,\left|y_{1}\right|<5 m$. Furthermore, we may use the same words and rules in order to create $w=x_{k+1} z y_{k+1}$ from $x_{1} z y_{1}$ by splicing. As $w$ does not belong to $L(I, S)$, the word $x_{1} z y_{1}$ must not belong to $L(I, S)$ either. If $z_{1}$ was in $I$, then $x_{1} z y_{1} \in I$ as well, as $z$ is at most as long as $\tilde{z}$.

Therefore, $z_{1}$ is created by a splicing $\left(w_{0}, w_{0}^{\prime}\right) \vdash_{s} z_{1}$ where $s=\left(u_{1}, u_{2} ; v\right), w_{0}=x u_{1}$, and $w_{0}^{\prime}=u_{2} y$ where $\left|u_{1}\right|,\left|u_{2}\right|<m$, by Lemma 3.4 (here, $x$ and $y$ are newly chosen words). We have

$$
z_{1}=x_{1} \tilde{z} y_{1}=x v y
$$

where $x$ is a proper prefix of $x_{1} \tilde{z}$ and $y$ is a proper suffix of $\tilde{z} y_{1}$. Recall that either $s \in S$ or $v=\mu$.

However, we will see next that if $v=\mu$, there is also a rule $\tilde{s} \in S$ and slightly modified words which can be used in order to create $x_{1} \tilde{z} y_{1}$ by splicing. In this case $\mu=\delta_{1} \alpha \beta \gamma \delta_{2}$ is a factor of $z_{1}$. As $\left|\delta_{1}\right|,\left|\delta_{2}\right| \geq 5 m>\left|x_{1}\right|,\left|y_{1}\right|$, the factor $\alpha \beta \gamma$ is covered by $\tilde{z}$ and, as such, the pumping algorithm ensured that either (a) $\alpha$ is succeeded by $\beta^{j / 2}$ or (b) $\gamma$ is preceded by $\beta^{j / 2}$. Due to symmetry, we only consider the former case, in which $\gamma \delta_{2}$ is a prefix of a word in $\beta^{+}$. Let us shorten the bridge $v$ such that $\tilde{s}=\left(u_{1}, u_{2} ; \delta_{1} \alpha \gamma \delta_{2}\right)$. Note that $\tilde{s} \in S$ (as $\alpha \sim \alpha \beta$ and by Lemma 3.3). Furthermore, as $j$ is large enough, $y=\beta_{2} \beta^{\ell} y^{\prime}$ where $\beta_{2}$ is the suffix of $\beta$ such that $\gamma \delta_{2} \beta_{2} \in \beta^{+}$and $\ell \geq|\gamma|$. Note that this implies $\beta_{2} \gamma$ is a prefix of $y$, which allows us to add an additional $\beta$. Therefore, $\left(w_{0}, u_{2} \beta_{2} \beta^{\ell+1} y^{\prime}\right) \vdash_{\tilde{s}} z_{1}$ where $u_{2} \beta_{2} \beta^{\ell+1} y^{\prime} \in L$. This observation justifies the assumption that $v \neq \mu$ and $s \in S$ which we will make for the remainder of the proof.

Next, we will pump down the factors $\alpha \beta^{j} \gamma$ to $\alpha \beta \gamma$ in $\tilde{z}$ again. At every position where we pumped up before, we are now pumping down (in reverse order) in order to obtain the words $\tilde{x}$, $\tilde{v}, \tilde{y}$ from the words $x, v, y$, respectively. The pumping in each step is done as in the proof of Lemma 3.9:

1. If $v$ overlaps with $\beta^{j}$ (in the factor that we are pumping down) but it neither covers $\alpha$ nor $\gamma$, extend $v$ (Lemma 3.2) such that $v=\alpha \beta^{j} \gamma$. Observe that, now, the factor $\alpha \beta^{j} \gamma$ is covered by either $x v$ or $v y$.

2. If $\alpha \beta^{j}$ or $\beta^{j} \gamma$ is covered by one of $x, v$, or $y$, then replace this factor by $\alpha \beta$ or $\beta \gamma$, respectively. Otherwise, by symmetry, assume that $\alpha \beta^{j} \gamma$ is covered by $x v$ and, therefore, we can factorize

$$
x=x^{\prime} \alpha \beta^{j_{1}} \beta_{1} \quad v=\beta_{2} \beta^{j_{2}} \gamma v^{\prime}
$$

where $\beta_{1} \beta_{2}=\beta$ and $j_{1}+j_{2}+1=j$. The results of pumping are the words $x^{\prime} \alpha \beta_{1}, \beta_{2} \gamma v^{\prime}$.

Let $\tilde{u}_{1}$ and $\tilde{u}_{2}$ be the sites of $s$ that may have been altered due to extensions and, by Lemma 3.4, assume $\left|\tilde{u}_{1}\right|,\left|\tilde{u}_{2}\right|<m$. If we used an extension for $v$ in at least one of the steps, then $|\tilde{v}| \leq m^{2}$. No matter whether or not we used an extension, $t=\left(\tilde{u}_{1}, \tilde{u}_{2} ; \tilde{v}\right) \in S$ and $\left(\tilde{x} \tilde{u}_{1}, \tilde{u}_{2} \tilde{y}\right) \vdash_{t} x_{1} z y_{1}$. As $\left|\tilde{x} \tilde{u}_{1}\right|,\left|\tilde{u}_{2} \tilde{y}\right|<|z|+6 m=|w|, \tilde{x} \tilde{u}_{1}$ and $\tilde{u}_{2} \tilde{y}$ belong to $L(I, S)$. We conclude that $x_{1} z y_{1}$ as well as $w$ belong to $L(I, S)$ - the desired contradiction.

\section{The Case of Classical Splicing}

In this section, we consider the splicing operation as defined in [18]. This is the most commonly used definition for splicing in formal language theory. The notation we use has been employed in previous papers, see e.g., [2,9]. Throughout this section, a quadruplet of words 
$r=\left(u_{1}, v_{1} ; u_{2}, v_{2}\right) \in\left(\Sigma^{*}\right)^{4}$ is called a (splicing) rule. The words $u_{1} v_{1}$ and $u_{2} v_{2}$ are called left and right site of $r$, respectively. This splicing rule can be applied to two words $w_{1}=x_{1} u_{1} v_{1} y_{1}$ and $w_{2}=x_{2} u_{2} v_{2} y_{2}$, that each contain one of the sites, in order to create the new word $z=x_{1} u_{1} v_{2} y_{2}$, see Figure 8. This operation is called splicing and it is denoted by $\left(w_{1}, w_{2}\right) \vdash_{r} z$. The splicing position of this splicing is $z\left[\left|x_{1} u_{1}\right|\right]$; that is the position between the factors $x_{1} u_{1}$ and $v_{2} y_{2}$ in $z$.

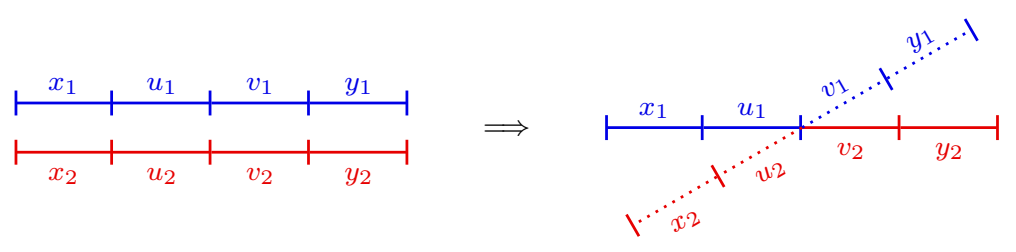

Figure 8: Splicing of the words $x_{1} u_{1} v_{1} y_{1}$ and $x_{2} u_{2} v_{2} y_{2}$ by the rule $r=\left(u_{1}, v_{1} ; u_{2}, v_{2}\right)$.

Just as in Section 3, for a rule $r$ we define the splicing operator $\sigma_{r}$ such that for a language $L$

$$
\sigma_{r}(L)=\left\{z \in \Sigma^{*} \mid \exists w_{1}, w_{2} \in L:\left(w_{1}, w_{2}\right) \vdash_{r} z\right\}
$$

and for a set of splicing rules $R$, we let

$$
\sigma_{R}(L)=\bigcup_{r \in R} \sigma_{r}(L) .
$$

The reflexive and transitive closure of the splicing operator $\sigma_{R}^{*}$ is given by

$$
\sigma_{R}^{0}(L)=L, \quad \sigma_{R}^{i+1}(L)=\sigma_{R}^{i}(L) \cup \sigma_{R}\left(\sigma_{R}^{i}(L)\right), \quad \sigma_{R}^{*}(L)=\bigcup_{i \geq 0} \sigma_{R}^{i}(L) .
$$

A finite set of axioms $I \subseteq \Sigma^{*}$ and a finite set of splicing rules $R \subseteq\left(\Sigma^{*}\right)^{4}$ form a splicing system $(I, R)$. Every splicing system $(I, R)$ generates a language $L(I, R)=\sigma_{R}^{*}(I)$. Note that $L(I, R)$ is the smallest language which is closed under the splicing operator $\sigma_{R}$ and includes $I$. It is known that the language generated by a splicing system is regular, see $[6,17]$. A (regular) language $L$ is called a splicing language if a splicing system $(I, R)$ exists such that $L=L(I, R)$.

A rule $r$ is said to respect a language $L$ if $\sigma_{r}(L) \subseteq L$. It is easy to see that for any splicing system $(I, R)$, every rule $r \in R$ respects the generated language $L(I, R)$. Moreover, a rule $r \notin R$ respects $L(I, R)$ if and only if $L(I, R \cup\{r\})=L(I, R)$. We say a splicing $\left(w_{1}, w_{2}\right) \vdash_{r} z$ respects a language $L$ if $w_{1}, w_{2} \in L$ and $r$ respects $L$; obviously, this implies $z \in L$, too.

The main result of this section states that, if a regular language $L$ is a splicing language, then it is generated by a particular splicing system $(I, R)$ which only depends on the syntactic monoid of $L$.

Theorem 4.1. Let $L$ be a splicing language and $m=\left|M_{L}\right|$. The splicing system $(I, R)$ with $I=\Sigma^{<m^{2}+6 m} \cap L$ and

$$
R=\left\{r \in \Sigma^{<m^{2}+10 m} \times \Sigma^{<2 m} \times \Sigma^{<2 m} \times \Sigma^{<m^{2}+10 m} \mid r \text { respects } L\right\}
$$

generates the language $L=L(I, R)$.

As the language generated by the splicing system $(I, R)$ is constructible, Theorem 4.1 implies that the problem whether or not a given regular language is a splicing language is decidable. A detailed discussion of the decidability result is given in Section 5 .

Let $L$ be a formal language. Clearly, every set of words $J \subseteq L$ and set of rules $S$ where every rule in $S$ respects $L$ generates a subset $L(J, S) \subseteq L$. Therefore, in Theorem 4.1 the inclusion $L(I, R) \subseteq L$ is obvious. The rest of this section is devoted to the proof of the converse inclusion $L \subseteq L(I, R)$. The proof uses many ideas that have been employed in the Section 3. However, 
there are some challenges we encounter solely while considering the classic splicing variant. The additional complexity comes from having to handle the first and fourth components of rules, which in the case of classical splicing occur both in the words used for splicing and the splicing result. In contrast, in Pixton splicing the sites of a rule do not occur in splicing result, whereas the bridge is not a factor of the words used for splicing. The structure of this section is the same as Section 3 . In Section 4.1 we will present techniques to obtain rules that respect a regular language $L$ from other rules that respect $L$, and we show how we can modify a splicing step, such that the words used for splicing are not significantly longer than the splicing result; similar results can be found in $[8,9]$. In Section 4.2 we use these techniques to show that a long word $z \in L$ can be obtained by a series of splicings from a set shorter words from $L$ and by using rules which satisfy certain length restrictions. Finally, in Section 4.3 we prove Theorem 4.1.

\subsection{Rule Modifications}

The first lemma states us that we can extend the sites of a rule $r$ such that the extended rule respects all languages that are respected by $r$.

Lemma 4.2. Let $r=\left(u_{1}, v_{1} ; u_{2}, v_{2}\right)$ be a rule which respects a language $L$. For every word $x$, the rules $\left(x u_{1}, v_{1} ; u_{2}, v_{2}\right),\left(u_{1}, v_{1} x ; u_{2}, v_{2}\right),\left(u_{1}, v_{1} ; x u_{2}, v_{2}\right)$, and $\left(u_{1}, v_{1} ; u_{2}, v_{2} x\right)$ respect $L$ as well.

Proof. Let $s$ be any of the rules $\left(x u_{1}, v_{1} ; u_{2}, v_{2}\right),\left(u_{1}, v_{1} x ; u_{2}, v_{2}\right),\left(u_{1}, v_{1} ; x u_{2}, v_{2}\right),\left(u_{1}, v_{1} ; u_{2}, v_{2} x\right)$. In order to prove that $s$ respects $L$, we have to show that, for all $w_{1}, w_{2} \in L$ and $z \in \Sigma^{*}$ such that $\left(w_{1}, w_{2}\right) \vdash_{s} z$, we have $z \in L$, too. Indeed, if $\left(w_{1}, w_{2}\right) \vdash_{s} z$, then $\left(w_{1}, w_{2}\right) \vdash_{r} z$ and, as $r$ respects $L$, we conclude $z \in L$.

Henceforth, for a rule $r=\left(u_{1}, v_{1} ; u_{2}, v_{2}\right)$, we will refer to the rules $\left(x u_{1}, v_{1} ; u_{2}, v_{2}\right)$ and $\left(u_{1}, v_{1} x ; u_{2}, v_{2}\right)$ as extensions of the left site of $r$ and to $\left(u_{1}, v_{1} ; x u_{2}, v_{2}\right)$ and $\left(u_{1}, v_{1} ; u_{2}, v_{2} x\right)$ as extensions of the right site of $r$.

Next, for a language $L$, let us investigate the syntactic class of a rule $r=\left(u_{1}, v_{1} ; u_{2}, v_{2}\right)$. The syntactic class (with respect to $L$ ) of $r$ is the set of rules $[r]_{L}=\left[u_{1}\right]_{L} \times\left[v_{1}\right]_{L} \times\left[u_{2}\right]_{L} \times\left[v_{2}\right]_{L}$ and two rules $r$ and $s$ are syntactically congruent (with respect to $L$ ), denoted by $r \sim_{L} s$, if $s \in[r]_{L}$.

Lemma 4.3. Let $r$ be a rule which respects a language $L$. Every rule $s \in[r]_{L}$ respects $L$.

Proof. Let $r=\left(u_{1}, v_{1} ; u_{2}, v_{2}\right)$ and $s=\left(\tilde{u}_{1}, \tilde{v}_{1} ; \tilde{u}_{2}, \tilde{v}_{2}\right)$. Thus, $u_{i} \sim_{L} \tilde{u}_{i}$ and $v_{i} \sim \tilde{v}_{i}$ for $i=1,2$. For $\tilde{w}_{1}=x_{1} \tilde{u}_{1} \tilde{v}_{1} y_{1} \in L$ and $\tilde{w}_{2}=x_{2} \tilde{u}_{2} \tilde{v}_{2} y_{2} \in L$ we have to show that $\tilde{z}=x_{1} \tilde{u}_{1} \tilde{v}_{2} y_{2} \in L$. For $i=1,2$, let $w_{i}=x_{i} u_{i} v_{i} y_{i}$ and note that $w_{i} \sim_{L} \tilde{w}_{i}$; hence, $w_{i} \in L$. Furthermore, $\left(w_{1}, w_{2}\right) \vdash_{r} x_{1} u_{1} v_{2} y_{2}=$ $z \in L$ as $r$ respects $L$, and $\tilde{z} \in L$ as $z \sim_{L} \tilde{z}$.

Consider a splicing $\left(x_{1} u_{1} v_{1} y_{1}, x_{2} u_{2} v_{2} y_{2}\right) \vdash_{r} x_{1} u_{1} v_{2} y_{2}$ which respects a regular language $L$, as shown in Figure 9 on the left site. The factors $v_{1} y_{1}$ and $x_{2} u_{2}$ may be relatively long but they do not occur as factors in the resulting word $x_{1} u_{1} v_{2} y_{2}$. In particular, it is possible that two long words are spliced and the outcome is a relatively short word. Using the Lemmas 4.2 and 4.3, we can find shorter words in $L$ and a modified splicing rule which can be used to obtain $x_{1} u_{1} v_{2} y_{2}$.

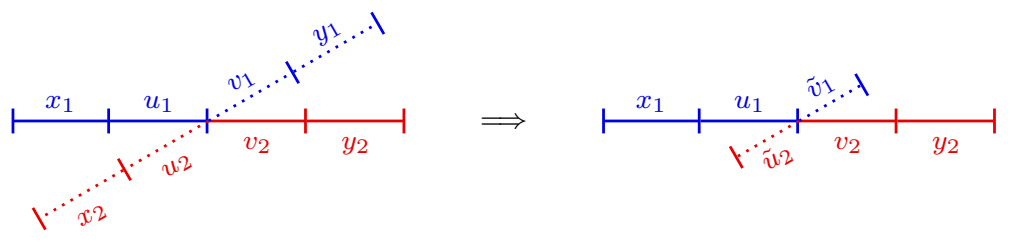

Figure 9: Replacing $v_{1} y_{1}$ and $x_{2} u_{2}$ by short words. 
Lemma 4.4. Let $r=\left(u_{1}, v_{1} ; u_{2}, v_{2}\right)$ be a rule which respects a regular language $L$ and $w_{1}=$ $x_{1} u_{1} v_{1} y_{1} \in L, w_{2}=x_{2} u_{2} v_{2} y_{2} \in L$. There is a rule $s=\left(u_{1}, \tilde{v}_{1} ; \tilde{u}_{2}, v_{2}\right)$ which respects $L$ and words $\tilde{w}_{1}=x_{1} u_{1} \tilde{v}_{1} \in L, \tilde{w}_{2}=\tilde{u}_{2} v_{2} y_{2} \in L$ such that $\left|\tilde{v}_{1}\right|,\left|\tilde{u}_{2}\right|<\left|M_{L}\right|$. More precisely, $\tilde{v}_{1} \in\left[v_{1} y_{1}\right]_{L}$ and $\tilde{u}_{2} \in\left[x_{2} u_{2}\right]_{L}$.

In particular, whenever $\left(w_{1}, w_{2}\right) \vdash_{r} x_{1} u_{1} v_{2} y_{2}=z$, then there is a splicing $\left(\tilde{w}_{1}, \tilde{w}_{2}\right) \vdash_{s} z$ which respects $L$ where $\tilde{w}_{1}, \tilde{w}_{2}$, and s have the properties described above.

Proof. By Lemma 4.2, the rule $\left(u_{1}, v_{1} y_{1} ; x_{2} u_{2}, v_{2}\right)$ respects $L$. Choose $\tilde{v}_{1} \in\left[v_{1} y_{1}\right]_{L}$ and $\tilde{u}_{2} \in$ $\left[x_{2} u_{2}\right]_{L}$ as shortest words from the sets, respectively. By Lemma 2.2, $\left|\tilde{u}_{1}\right|,\left|\tilde{u}_{2}\right|<\left|M_{L}\right|$ and $\tilde{w}_{1}=x_{1} u_{1} \tilde{v}_{1} \in L, \tilde{w}_{2}=\tilde{u}_{2} v_{2} y_{2} \in L$. Furthermore, by Lemma $4.3, s=\left(u_{1}, \tilde{v}_{1} ; \tilde{u}_{2}, v_{2}\right)$ respects L.

\subsection{Series of Splicings}

Consider the creation of words by a series of splicings. Let us begin with a simple observation. In the case when a word is created by two (or more) successive splicings, but none of the splicing sites overlaps the position of the other splicing, the order of these splicings is irrelevant. Recall that the splicing position of a splicing $\left(w_{1}, w_{2}\right) \vdash_{r} z$ with $r=\left(u_{1}, v_{1} ; u_{2}, v_{2}\right)$ is the position between the factors $u_{1}$ and $v_{2}$ in $z$. The notation in Remark 4.5 is the same as in the Figure 10.

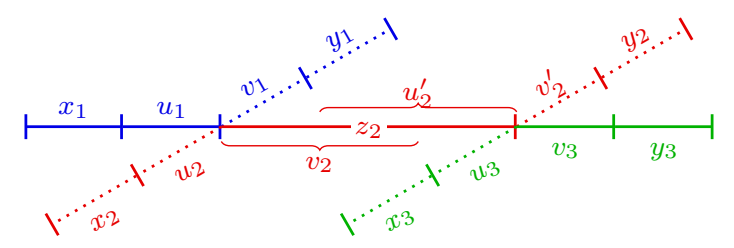

Figure 10: The word $x_{1} u_{1} z_{2} v_{3} y_{3}$ can be created either by using the right splicing first or by using the left splicing first.

Remark 4.5. Let $w_{1}=x_{1} u_{1} v_{1} y_{1}, w_{2}=x_{2} u_{2} z_{2} v_{2}^{\prime} y_{2}$, where $v_{2}$ is a prefix of $z_{2}$ and $u_{2}^{\prime}$ is a suffix of $z_{2}, w_{3}=x_{3} u_{3} v_{3} y_{3}$ be words and $r_{1}=\left(u_{1}, v_{1} ; u_{2}, v_{2}\right), r_{2}=\left(u_{2}^{\prime}, v_{2}^{\prime} ; u_{3}, v_{3}\right)$ be rules. In order to create the word $z=x_{1} u_{1} z_{2} v_{3} y_{3}$ by splicing, we may use splicings

$$
\begin{array}{llr}
\left(w_{1}, w_{2}\right) \vdash_{r_{1}} x_{1} u_{1} z_{2} v_{2}^{\prime} y_{2}=z^{\prime}, & \left(z^{\prime}, w_{3}\right) \vdash_{r_{2}} z & \text { or } \\
\left(w_{2}, w_{3}\right) \vdash_{r_{2}} x_{2} u_{2} z_{2} v_{3} y_{3}=z^{\prime \prime}, & \left(w_{1}, z^{\prime \prime}\right) \vdash_{r_{1}} z .
\end{array}
$$

Now, consider a word $z$ which is created by two successive splicings from words $w_{i}=x_{i} u_{i} v_{i} y_{i}$ for $i=1,2,3$ as in Figure 11. If no factor of $w_{1}$ is a part of $z$, then we can find another splicing rule $s$ such that $\left(w_{3}, w_{2}\right) \vdash_{s} z$. This replacement will become crucial in the proof of Lemma 4.7.

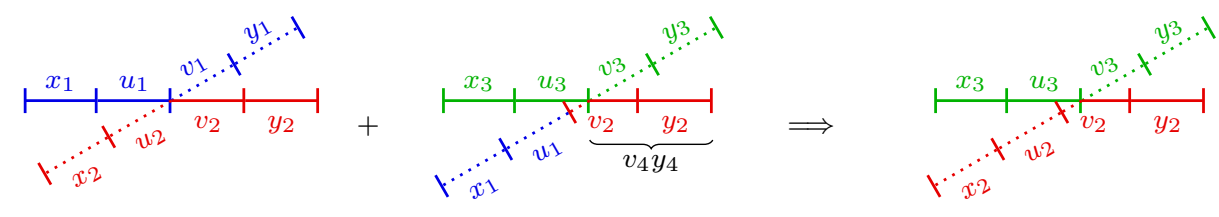

Figure 11: If no part of $x_{1} u_{1} v_{1} y_{1}$ is a factor of the splicing result, then the two splicings can be reduced to one splicing.

Lemma 4.6. Let $L$ be a language, $w_{i}=x_{i} u_{i} v_{i} y_{i} \in L$ for $i=1,2,3$, and $r_{1}=\left(u_{1}, v_{1} ; u_{2}, v_{2}\right)$, $r_{2}=\left(u_{3}, v_{3} ; u_{4}, v_{4}\right)$ be rules respecting $L$. If there are splicings

$$
\left(w_{1}, w_{2}\right) \vdash_{r_{1}} x_{1} u_{1} v_{2} y_{2}=w_{4}=x_{4} u_{4} v_{4} y_{4}, \quad\left(w_{3}, w_{4}\right) \vdash_{r_{2}} x_{3} u_{3} v_{4} y_{4}=z
$$


where $v_{4} y_{4}$ is a suffix of $v_{2} y_{2}$, then there is a rule $s=\left(u_{3}, v_{3} ; u_{2} \delta, \tilde{v}_{4}\right)$ which respects $L$ and $\left(w_{3}, w_{2}\right) \vdash_{s} z$. Furthermore, $\tilde{v}_{4}=v_{4}$ or $\tilde{v}_{4} \leq_{\ell \ell} v_{2}$.

Proof. Extension (Lemma 4.2) justifies the assumption that the factors $u_{1} v_{2}$ and $u_{4} v_{4}$ match in $w_{4}$ : let $w_{4}[i, j]=u_{1} v_{2}$ and $w_{4}\left[i^{\prime}, j^{\prime}\right]=u_{4} v_{4}$,

- if $i<i^{\prime}$ we extend $u_{4}$ in $r_{2}$ to the left by $i^{\prime}-i$ letters,

- if $i>i^{\prime}$ we extend $u_{1}$ in $r_{1}$ to the left by $i-i^{\prime}$ letters,

- if $j<j^{\prime}$ we extend $v_{2}$ in $r_{1}$ to the right by $j^{\prime}-j$ letters, and

- if $j>j^{\prime}$ we extend $v_{4}$ in $r_{2}$ to the right by $j-j^{\prime}$ letters.

Clearly, the extended factors $u_{1} v_{2}$ and $u_{4} v_{4}$ match in $w_{4}$. As $v_{4} y_{4}$ was a suffix of $v_{2} y_{2}$ before extension, now, $v_{4}$ is a suffix of $v_{2}$ and $y_{2}=y_{4}$. Additionally, either $v_{4}$ was not extended or $v_{4} \leq_{\ell \ell} v_{2}$ and $v_{2}$ was not extended. Let $\delta$ such that $\delta v_{4}=v_{2}$, let $s=\left(u_{3}, v_{3} ; u_{2} \delta, v_{4}\right)$, and observe that $\left(w_{3}, w_{2}\right) \vdash_{s} z$.

Next, let us prove that $s$ respects $L$. Let $w_{3}^{\prime}=x_{3}^{\prime} u_{3} v_{3} y_{3}^{\prime} \in L$ and $w_{2}^{\prime}=x_{2}^{\prime} u_{2} \delta v_{4} y_{2}^{\prime}=x_{2}^{\prime} u_{2} v_{2} y_{2}^{\prime} \in$ $L$. There are splicings

$$
\left(w_{1}, w_{2}^{\prime}\right) \vdash_{r_{1}} x_{1} u_{1} v_{2} y_{2}^{\prime}=w_{4}^{\prime}=x_{1} u_{4} v_{4} y_{2}^{\prime}, \quad\left(w_{3}^{\prime}, w_{4}^{\prime}\right) \vdash_{r_{2}} x_{3}^{\prime} u_{3} v_{4} y_{2}^{\prime}=z^{\prime}
$$

and $z^{\prime} \in L$, concluding that $s$ respects $L$.

Consider a splicing system $(J, S)$ and its generated language $L=L(J, S)$. Let $n$ be the length of the longest word in $J$ and let $\mu$ be the length-lexicographically largest word that is a component of a rule in $S$. Define $W_{\mu}=\left\{w \in \Sigma^{*} \mid w \leq_{\ell \ell} \mu\right\}$ as the set of words which are at most as large as $\mu$, in length-lexicographic order. Furthermore, let $I=\Sigma^{\leq n} \cap L$ be a set of axioms and let

$$
R=\left\{r \in W_{\mu}^{4} \mid r \text { respects } L\right\}
$$

be a set of rules. It is not difficult to see that $J \subseteq I, S \subseteq R$, and $L=L(I, R)$. Whenever convenient, we will assume that a splicing language $L$ is generated by a splicing system which is of the form of $(I, R)$.

Now, let us consider a word $x z y \in L$ where the length of the middle factor $z$ is at least $|\mu|$. The creation of $x z y$ by splicing in $(I, R)$ can be traced back to a word $x_{1} z y_{1}=z_{1}$ where either $z_{1} \in I$ or where $z_{1}$ is created by a splicing that affects the factor $z$, i. e., the splicing position lies in the factor $z$. The next lemma describes this creation of $x z y=z_{k+1}$ by $k$ splicings in $(I, R)$, and shows that we can choose the rules and words which are used to create $z_{k+1}$ from $z_{1}$ such that the words are not significantly longer than $\ell=\max \{|x|,|y|\}$ and such that the rules satisfy certain length restrictions.

Lemma 4.7. Let $L$ be a splicing language, let $\ell, n \in \mathbb{N}$, let $m=\left|M_{L}\right|$, and let $\mu$ be a word with $|\mu| \geq \ell+2 m$ such that for $I=\Sigma^{\leq n} \cap L$ and $R=\left\{r \in W_{\mu}^{4} \mid r\right.$ respects $\left.L\right\}$ we have $L=L(I, R)$.

Let $z_{k+1}=x_{k+1} z y_{k+1}$ with $|z| \geq|\mu|$ and $\left|x_{k+1}\right|,\left|y_{k+1}\right| \leq \ell$ be a word that is created by $k$ splicings from a word $z_{1}=x_{1} z y_{1}$ where either $z_{1} \in I$ or $z_{1}$ is created by a splicing $\left(w_{0}, w_{0}^{\prime}\right) \vdash_{s} z_{1}$ where $w_{0}, w_{0}^{\prime} \in L, s$ respects $L$, and the splicing position lies in the factor $z$. Furthermore, for $i=1, \ldots, k$ the intermediate splicings are either

(i) $\left(w_{i}, z_{i}\right) \vdash_{r_{i}} x_{i+1} z y_{i+1}=z_{i+1}, w_{i} \in L, r_{i} \in R, y_{i+1}=y_{i}$, and the splicing position lies at the left of the factor $z$ or

(ii) $\left(z_{i}, w_{i}\right) \vdash_{r_{i}} x_{i+1} z y_{i+1}=z_{i+1}, w_{i} \in L, r_{i} \in R, x_{i+1}=x_{i}$, and the splicing position lies at the right of the factor $z$.

There are rules and words creating $z_{k+1}$, as above, satisfying in addition:

1. There is $k^{\prime} \leq k$ such that for $i=1, \ldots, k^{\prime}$ all splicings are of the form (i) and for $i=$ $k^{\prime}+1, \ldots, k$ all splicings are of the form (ii). 
2. For $i=1, \ldots, k^{\prime}$ the following bounds apply: $\left|x_{i}\right|<\ell+2 m,\left|w_{i}\right|<\ell+2 m, r_{i} \in \Sigma^{<\ell+m} \times$ $\Sigma^{<2 m} \times \Sigma^{<2 m} \times W_{\mu}$, and $x_{k^{\prime}+1}=x_{k^{\prime}+2}=\cdots=x_{k+1}$.

3. For $i=k^{\prime}+1, \ldots, k$ the following bounds apply: $\left|y_{i}\right|<\ell+2 m,\left|w_{i}\right|<\ell+2 m, r_{i} \in$ $W_{\mu} \times \Sigma^{<2 m} \times \Sigma^{<2 m} \times \Sigma^{<\ell+m}$, and $y_{1}=y_{2}=\cdots=y_{k^{\prime}+1}$.

In particular, if $n \geq \ell+2 m$, then $w_{1}, \ldots, w_{k} \in I$.

Proof. The first statement follows immediately by Remark 4.5 and the fact that $|z| \geq|\mu|$. The first statement also implies implies $x_{k^{\prime}+1}=x_{k^{\prime}+2}=\cdots=x_{k+1}$ and $y_{1}=y_{2}=\cdots=y_{k^{\prime}+1}$. Note that if $k^{\prime}=0\left(\right.$ or $\left.k^{\prime}=k\right)$, then statement 2 (resp. statement 3 ) is trivially true.

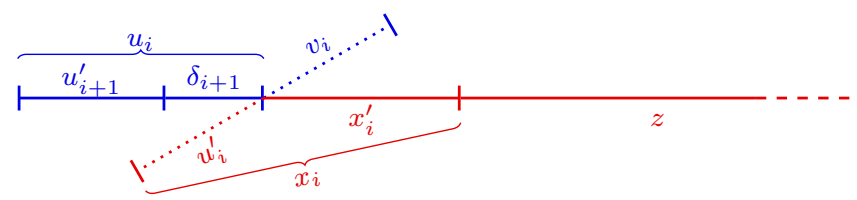

Figure 12: The $i$-th splicing for $i \leq k^{\prime}$ in the proof of Lemma 4.7 where $x_{i+1}=u_{i} x_{i}^{\prime}$ and $v_{i}^{\prime}$ is a prefix of $x_{i}^{\prime} z$.

The notation we employ in order to prove statement 2 is chosen such that it matches with Figure 12. For $i=1, \ldots, k^{\prime}$, let $r_{i}=\left(u_{i}, v_{i} ; u_{i}^{\prime}, v_{i}^{\prime}\right)$. By extension (Lemma 4.2), we may assume that $w_{i}=u_{i} v_{i}$ and $x_{i}=u_{i}^{\prime} x_{i}^{\prime}$ such that $x_{i+1}=u_{i} x_{i}^{\prime}$ and $v_{i}^{\prime}$ is a prefix of $x_{i}^{\prime} z$. Let $x_{k^{\prime}+1}^{\prime}=x_{k^{\prime}+1}=x_{k+1}$ and $u_{k^{\prime}+1}^{\prime}=\varepsilon$. By Lemma 4.6, we may assume that every splicing position lies at the left of the previous splicing position, i. e., $x_{i}^{\prime}$ is a proper suffix of $x_{i+1}^{\prime}$ and $\left|x_{i}^{\prime}\right| \leq \ell$ as $\left|x_{k^{\prime}+1}^{\prime}\right| \leq \ell$. Due to the modifications we made, we may have lost control of the lengths of $u_{i}$, $v_{i}$, and $u_{i}^{\prime}$; but $v_{i}^{\prime}$ still belongs to $W_{\mu}$ and $r_{i}$ respects $L$. Let $\delta_{i+1}$ such that $x_{i+1}^{\prime}=\delta_{i+1} x_{i}^{\prime}$; hence, $u_{i}=u_{i+1}^{\prime} \delta_{i+1}$. The factor $\delta_{i+1}$ is the the part of $x_{k+1}$ which is added by the $i$-th splicing and is not modified afterwards; $x_{k+1}=\delta_{k^{\prime}+1} \cdots \delta_{2} x_{1}^{\prime}$. Now, for $i=2, \ldots, k^{\prime}$, we replace $u_{i}^{\prime}$ by a shortest word from $\left[u_{i}^{\prime}\right]_{L}$. (We also replace this prefix of $x_{i}$ and $u_{i-1}$.) Furthermore, we replace $v_{i}$ by a shortest word from $\left[v_{i}\right]_{L}$ for $i=1, \ldots, k^{\prime}$. By Lemma 2.2, we have $\left|u_{i}^{\prime}\right|,\left|v_{i}\right|<m$. We do not replace $u_{1}^{\prime}$ yet, as this might affect the word $w_{0}$ and the rule $s$ in the splicing $\left(w_{0}, w_{0}^{\prime}\right) \vdash_{s} x_{1} z y_{1}$.

Observe that the words $z_{i}, w_{i}$, and the rules $r_{i}$ can still be used to create $z_{k+1}$ by splicing, in the way described in the claim. For $i=2, \ldots, k^{\prime}$, we have $\left|x_{i}\right|=\left|u_{i}^{\prime} x_{i}^{\prime}\right|<\ell+m,\left|w_{i}\right| \leq$ $\left|x_{i+1}\right|+\left|v_{i}\right|<\ell+2 m$, and $r_{i} \in \Sigma^{<\ell+m} \times \Sigma^{<m} \times \Sigma^{<m} \times W_{\mu}$. We also have $\left|w_{1}\right|<\ell+2 m$ and $r_{1} \in \Sigma^{<\ell+m} \times \Sigma^{<m} \times \Sigma^{*} \times W_{\mu}$. Note that, except for the length of $x_{1}$, and the third component of $r_{1}$, we have proven statement 2 (of the lemma) and we actually have proven a stronger bound than claimed. Symmetrically, we can consider statement 3 to be proven except for $y_{1}=y_{k^{\prime}+1}$ and the second component of $r_{k^{\prime}+1}$.

Let $x_{1}=u_{1}^{\prime} x_{1}^{\prime}$ as above and, symmetrically, let $y_{1}=y_{k^{\prime}+1}^{\prime} v_{k^{\prime}+1}^{\prime}$ where $v_{k^{\prime}+1}^{\prime}$ is the second component of $r_{k^{\prime}+1}$. If $k^{\prime}=0$ (or $k^{\prime}=k$ ), then $u_{1}^{\prime}\left(\right.$ resp. $v_{k^{\prime}+1}^{\prime}$ ) can be considered empty and $x_{1}^{\prime}=x_{k+1}\left(\operatorname{resp} . y_{k^{\prime}+1}^{\prime}=y_{k+1}\right)$. If $z_{1} \in I$, we replace $u_{1}^{\prime}$ and $v_{k^{\prime}+1}^{\prime}$ by shortest words from their syntactic classes, respectively, and the claim holds by Lemma 2.2. Otherwise, $\left(w_{0}, w_{0}^{\prime}\right) \vdash_{s} z_{1}$ where $u_{1}^{\prime}$ is a prefix of $w_{0}$ and $v_{k^{\prime}+1}^{\prime}$ is a suffix of $w_{0}^{\prime}$.

Let $s=\left(u_{0}, v_{0} ; u_{0}^{\prime}, v_{0}^{\prime}\right)$ and consider the overlap of the factor $u_{0}$ in the splicing $\left(w_{0}, w_{0}^{\prime}\right) \vdash_{s} z_{1}$ with the prefix $u_{1}^{\prime}$ of $w_{0}$. In case when $u_{0}$ does not overlap with $u_{1}^{\prime}$, replace $u_{1}^{\prime}$ by a shortest word from its syntactic class. If $u_{0}$ and $u_{1}^{\prime}$ overlap, let $u_{1}^{\prime}=\delta_{1} \delta_{2}$ such that $\delta_{2}$ is the overlap and replace $\delta_{1}$ and $\delta_{2}$ by shortest words from their syntactic classes, respectively. Note that if we modified $u_{1}$, it got shorter; hence, $s$ still belongs to $R$. In any case, $\left|u_{1}^{\prime}\right|<2 m,\left|x_{1}\right|<\ell+2 m$ (Lemma 2.2), and $r_{1} \in \Sigma^{<\ell+m} \times \Sigma^{<m} \times \Sigma^{<2 m} \times W_{\mu}$; thus, the second statement.

We may treat $v_{k^{\prime}+1}^{\prime}$ and $r_{k^{\prime}+1}$ symmetrically in order to prove statement 3 . 


\subsection{Proof of Theorem 4.1}

Let $L$ be a splicing language and $m=\left|M_{L}\right|$. Throughout this section, by $\sim$ we denote the equivalence relation $\sim_{L}$ and by $[\cdot]$ we denote the corresponding equivalence classes $[\cdot]_{L}$.

Recall that Theorem 4.1 claims that the splicing system $(I, R)$ with $I=\Sigma^{<m^{2}+6 m} \cap L$ and

$$
R=\left\{r \in \Sigma^{<m^{2}+10 m} \times \Sigma^{<2 m} \times \Sigma^{<2 m} \times \Sigma^{<m^{2}+10 m} \mid r \text { respects } L\right\}
$$

generates $L$. The proof is divided in two parts. In the first part, Lemma 4.8, we prove that the set of rules can be chosen as $\left\{r \in\left(\Sigma^{<m^{2}+10 m}\right)^{4} \mid r\right.$ respects $\left.L\right\}$ for some finite set of axioms. The second part concludes the proof of Theorem 4.1, by employing the length bound $2 m$ for the second and third component of rules and by proving that the set of axioms can be chosen as $I=\Sigma^{<m^{2}+6 m} \cap L$.

Lemma 4.8. Let $L$ and $m$ as above. There exists $n \in \mathbb{N}$ such that the splicing system $(I, R)$ with $I=\Sigma^{\leq n} \cap L$ and

$$
R=\left\{r \in\left(\Sigma^{<m^{2}+10 m}\right)^{4} \mid r \text { respects } L\right\}
$$

generates the same language $L=L(I, R)$.

Proof. As every word in $I$ belongs to $L$ and every rule in $R$ respects $L$, the inclusion $L(I, R) \subseteq L$ holds (for any $n$ ).

Since $L$ is a splicing language, there exists a splicing system $\left(I^{\prime}, R^{\prime}\right)$ which generates $L$. Let $n^{\prime}$ be a number larger than any word in $I^{\prime}$ and larger than any component of a rule in $R^{\prime}$ and let $n=n^{\prime}+6 m$. Let $I=\Sigma^{\leq n} \cap L$ as in the claim and observe that $L\left(I, R^{\prime}\right)=L$.

For a word $\mu$ we let $W_{\mu}=\left\{w \in \Sigma^{*} \mid w \leq_{\ell \ell} \mu\right\}$, as we did before. Define the set of rules where every component is length-lexicographically bounded by $\mu$

$$
R_{\mu}=\left\{r \in W_{\mu}^{4} \mid r \text { respects } L\right\}
$$

and the language $L_{\mu}=L\left(I, R_{\mu}\right)$; clearly, $L_{\mu} \subseteq L$. For two words $\mu \leq_{\ell \ell} v$ we see that $R_{\mu} \subseteq R_{v}$, and hence, $L_{\mu} \subseteq L_{v}$. Thus, if $L_{\mu}=L$ for some word $\mu$, then for all words $v$ with $\mu \leq_{\ell \ell} v$, we have $L_{v}=L$. As $L=L\left(I, R^{\prime}\right)$, there exists a word $\mu$ such that $L_{\mu}=L$ and $|\mu|+6 m \leq n$. Let $\mu$ be the smallest word, in the length-lexicographic order, such that $L_{\mu}=L$. Note that if $|\mu|<m^{2}+10$, then $R_{\mu} \subseteq R$ and $L=L_{\mu} \subseteq L(I, R)$. For the sake of contradiction assume $|\mu| \geq m^{2}+10 m$. Let $\nu$ be the next-smaller word than $\mu$, in the length-lexicographic order, and let $S=R_{\nu}$. Note that $L(I, S) \subsetneq L$ and $R_{\mu} \backslash S$ contains only rules which have a component that is equal to $\mu$.

Choose $w$ from $L \backslash L(I, S)$ as a shortest word, i.e., for all $w^{\prime} \in L$ with $\left|w^{\prime}\right|<|w|$, we have $w^{\prime} \in L(I, S)$. Factorize $w=x z y$ with $|x|=|y|=3 m$ and note that $|z| \geq|\mu|$, otherwise $w \in I$. Factorize $\mu=\delta_{1} \alpha \beta \gamma \delta_{2}$ with $\left|\delta_{1}\right|,\left|\delta_{2}\right| \geq 5 m,|\alpha \beta \gamma|=m^{2}, \beta \neq \varepsilon, \alpha \sim \alpha \beta$, and $\gamma \sim \beta \gamma$ (Lemma 2.1).

We will show that there is a series of splicings which creates $w$ from a set of shorter words and by using splicing rules from $S$. This yields a contradiction to the choice of $w$. In order to find this series of splicings we investigate the creation of a word $x \tilde{z} y$ where $\tilde{z}$ is derived by using a pumping argument on all factors $\alpha \beta \gamma$ in $z$.

Let $j$ be a sufficiently large even number $(j>4|\mu|+|z|$ will suffice $)$. We define a word $\tilde{z}$ which is the result of applying the pumping algorithm from Lemma 2.3 on $z$, as discussed in Section 2.1. The pumping algorithm replaces the occurrences of $\alpha \beta \gamma$ in $z$ by $\alpha \beta^{j} \gamma$ such that for every factor $\tilde{z}\left[k, k+m^{2}\right]=\alpha \beta \gamma$, either

(a) $\alpha \beta^{j / 2}$ is a factor of $\tilde{z}$ starting at position $\tilde{z}[k]$ or

(b) $\beta^{j / 2} \gamma$ is a factor of $\tilde{z}$ ending at position $\tilde{z}\left[k+m^{2}\right]$

holds. In particular, if $\delta_{1} \alpha \beta \gamma \delta_{2}$ is a factor of $\tilde{z}$ either (a) $\gamma \delta_{2}$ is a prefix of a word in $\beta^{+}$or (b) $\delta_{1} \alpha$ is a suffix of a word in $\beta^{+}$. By induction and as $\alpha \beta \gamma \sim \alpha \beta^{j} \gamma$, it is easy to see that $z \sim \tilde{z}$ and $x \tilde{z} y \in L$. 
Let us trace back the creation of $x \tilde{z} y \in L$ by splicing in $\left(I, R_{\mu}\right)$ to a word $x_{1} \tilde{z} y_{1}$ where either $x_{1} \tilde{z} y_{1} \in I$ or where $x_{1} \tilde{z} y_{1}$ is created by a splicing that affects $\tilde{z}$, i. e., the splicing position lies within the factor $\tilde{z}$. Let $z_{k+1}=x_{k+1} \tilde{z} y_{k+1}$, where $x_{k+1}=x$ and $y_{k+1}=y$, be created by $k$ splicings from a word $z_{1}=x_{1} \tilde{z} y_{1}$ where either $x_{1} \tilde{z} y_{1} \in I$ or $x_{1} \tilde{z} y_{1}$ is created by a splicing $\left(w_{0}, w_{0}^{\prime}\right) \vdash_{s} z_{1}$ with $w_{0}, w_{0}^{\prime} \in L, s \in R_{\mu}$, and the splicing position lies in the factor $\tilde{z}$. Furthermore, for $i=1, \ldots, k$ the intermediate splicings are either

(i) $\left(w_{i}, z_{i}\right) \vdash_{r_{i}} x_{i+1} \tilde{z} y_{i+1}=z_{i+1}, w_{i} \in L, r_{i} \in R_{\mu}, y_{i+1}=y_{i}$, and the splicing position lies at the left of the factor $\tilde{z}$ or

(ii) $\left(z_{i}, w_{i}\right) \vdash_{r_{i}} x_{i+1} \tilde{z} y_{i+1}=z_{i+1}, w_{i} \in L, r_{i} \in R_{\mu}, x_{i+1}=x_{i}$, and the splicing position lies at the right of the factor $\tilde{z}$.

Note that $|\tilde{z}| \geq|z| \geq|\mu|$ and, therefore, we can apply Lemma 4.7 (with $\ell=3 m$ ). Thus, we may assume that $w_{i} \in I$ and $\left|x_{i}\right|,\left|y_{i}\right|<5 m$ for $i=1, \ldots, k$.

Consider a rule $r_{i}$ in a splicing of the form (i). By Lemma 4.7, $r_{i} \in \Sigma^{<4 m} \times \Sigma^{<2 m} \times \Sigma^{<2 m} \times W_{\mu}$. Suppose the fourth component of $r_{i}$ covers a prefix of the left-most factor $\alpha \beta^{j / 2}$ in $\tilde{z}$ which is longer than $\alpha$ (as $j$ is very large, it cannot fully cover $\alpha \beta^{j / 2}$ ). By extension (Lemma 4.2), we may write $r_{i}=\left(u_{1}, v_{1} ; u_{2}, v^{\prime} \alpha \beta^{h}\right)$ for some $h \geq 1$. By Lemma 4.3 and as $\alpha \sim \alpha \beta$, we may replace this rule by $\left(u_{1}, v_{1} ; u_{2}, v^{\prime} \alpha\right)$. Note that, as the fourth component got shorter, now $r_{i} \in S$.

After we symmetrically treated rules of form (ii), these new rules $r_{1}, \ldots, r_{k}$ and the words $w_{1}, \ldots, w_{k}$ can be used in order to create $w=x_{k+1} z y_{k+1}$ from $x_{1} z y_{1}$ by splicing. In order to see this, observe that, even though the factors $\alpha \beta \gamma$ in $z$, which we pumped up before, may overlap with each other, the left-most (and right-most) position where we replaced $\beta$ by $\beta^{j}$ is preceded by the factor $\alpha$ (resp. succeeded by the factor $\gamma$ ) in $\tilde{z}$.

Next, we show that all the rules $r_{1}, \ldots, r_{k}$ belong to $S$, now. By contradiction, suppose $r_{i} \notin S$ for some $i$ and, by symmetry, suppose this $i$-th splicing is of the form (i). Thus, the fourth component of $r_{i}$ has to be $\mu=\delta_{1} \alpha \beta \gamma \delta_{2}$. As $\left|\delta_{1}\right| \geq 5 m>\left|x_{i}\right|$, the factor $\alpha \beta \gamma$ in $\mu$ is covered by $\tilde{z}$. Let $k$ such that $\alpha \beta \gamma=\tilde{z}\left[k ; k+m^{2}\right]$ is this factor in $\tilde{z}$. The pumping algorithm ensured that (a) $\alpha \beta^{j / 2}$ is a factor of $\tilde{z}$ starting at position $\tilde{z}[k]$ or (b) $\beta^{j / 2} \gamma$ is a factor of $\tilde{z}$ ending at position $\tilde{z}\left[k+m^{2}\right]$. As $j / 2$ is very large and the splicing position of $\left(w_{i}, z_{i}\right) \vdash_{r_{i}} z_{i+1}$ is too close to the left end of $z_{i+1}$, case (b) is not possible. Thus, case (a) holds, the fourth component of $r_{i}$ overlaps in more than $|\alpha|$ letters with the left-most factor $\alpha \beta^{j / 2}$ in $\tilde{z}$, and we used the replacement above which ensured $r_{i} \in S$ - contradiction.

Let us summarize: if $x_{1} z y_{1}$ was in $L(I, S)$, then $w \in L(I, S)$ as well, which would contradict the choice of $w$. If $z_{1}=x_{1} \tilde{z} y_{1} \in I$, then $x_{1} z y_{1}$, which is at most as long as $z_{1}$, would belong to $I$ and we are done. We only have to consider the case when $\left(w_{0}, w_{0}^{\prime}\right) \vdash_{s} z_{1}=x_{1} \tilde{z} y_{1}$ and the splicing position lies within the factor $\tilde{z}$. We will show that, from this splicing, we derive another splicing $\left(\tilde{w}_{0}, \tilde{w}_{0}^{\prime}\right) \vdash_{t} x_{1} z y_{1}$ which respects $L(I, S)$ and, therefore, yields the contradiction.

Let $s=\left(u, v_{1} ; u_{2}, v\right), w_{0}=x u v_{1}$ and $w_{0}^{\prime}=u_{2} v y$ where $\left|v_{1}\right|,\left|u_{2}\right|<m$, by Lemma 4.4 (here, $x$ and $y$ are newly chosen words). We have

$$
z_{1}=x_{1} \tilde{z} y_{1}=x u v y
$$

where $x u$ is a proper prefix of $x_{1} \tilde{z}$ and $v y$ is a proper suffix of $\tilde{z} y_{1}$.

We will see next that if $s \notin S$, then we can use a rule $\tilde{s} \in S$ and maybe slightly modified words in order to obtain $z_{1}$ by splicing. If $s \notin S$, then $u=\mu$ or $v=\mu$. Suppose $u=\mu=\delta_{1} \alpha \beta \gamma \delta_{2}$. Thus, the factor $\alpha \beta \gamma$ of $\mu$ is covered by the factor $\tilde{z}$ in $z_{1}$ as $\left|\delta_{1}\right| \geq 5 m>\left|x_{1}\right|$. Let $\alpha \beta \gamma=\tilde{z}\left[k ; k+m^{2}\right]$ be this factor. (a) $\alpha \beta^{j / 2}$ is a factor of $\tilde{z}$ starting at position $\tilde{z}[k]$ or (b) $\beta^{j / 2} \gamma$ is a factor of $\tilde{z}$ ending at position $\tilde{z}\left[k+m^{2}\right]$. If (b) holds, $\delta_{1} \alpha$ is a suffix of a word in $\beta^{+}$. We may write $\delta_{1} \alpha=\beta_{2} \beta^{\ell}$ where $\ell \geq 0$ and $\beta_{2}$ is a suffix of $\beta$. Replace $u$ by $\beta_{2} \gamma \delta_{1}$ and use this new rule $\tilde{s}$ in order to splice $\left(w_{0}, w_{0}^{\prime}\right) \vdash_{\tilde{s}} z_{1}$. Note that the first component is now shorter than $\mu$. Otherwise, (a) holds and $\gamma \delta_{2} v$ is a prefix of a word in $\beta^{+}$. As $j$ is very large and $\gamma$ is a prefix of a word in $\beta^{+}$, we may extend $v$ (Lemma 4.2) such that we can write $\beta \gamma \delta_{2}=\beta^{\ell_{1}} \beta_{1}$ and $v=\beta_{2} \beta^{\ell_{2}} \gamma$ where $\ell_{1} \geq 1, \ell_{2} \geq 0$ and $\beta_{1} \beta_{2}=\beta$. Now, we pump down one of the $\beta$ in the first component and $\beta^{\ell_{2}}$ in the fourth 
component and we let $\tilde{s}=\left(\delta_{1} \alpha \beta^{\ell_{1}-1} \beta_{1}, v_{1} ; u_{2}, \beta_{2} \gamma\right) \sim s$. As all components are shorter than $\mu$, we see that $\tilde{s} \in S$ and

$$
\left(x \delta_{1} \alpha \beta^{\ell_{1}-1} \beta_{1} v_{1}, u_{2} \beta_{2} \beta^{\ell_{2}+1} \gamma y\right) \vdash_{\tilde{s}} z_{1},
$$

i.e., we have shifted one of the occurrences of $\beta$ from $w_{0}$ to $w_{0}^{\prime}$. Note that $\beta_{2} \gamma$ is a prefix of $\beta_{2} \beta^{\ell_{2}+1} \gamma$. Treating the fourth component analogously justifies the assumption that $s \in S$.

Next, we will pump down the factors $\alpha \beta^{j} \gamma$ to $\alpha \beta \gamma$ in $\tilde{z}$ again. At every position where we pumped up before, we are now pumping down (in reverse order) in order to obtain the words $\tilde{x}, \tilde{u}, \tilde{v}, \tilde{y}$ from the words $x, u, v, y$, respectively. For each pumping step do:

1. If $u$ is covered by the factor $\alpha \beta^{j} \gamma$ (which we pump down in this step), extend $u$ to the left such that it becomes a prefix of $\alpha \beta^{j} \gamma$. Symmetrically, if $v$ is covered by the factor $\alpha \beta^{j} \gamma$, extend $v$ to the right such that it becomes a suffix of $\alpha \beta^{j} \gamma$ (Lemma 4.2). Observe that extension ensures that the factor $\alpha \beta^{j} \gamma$ is covered by either $x u, u v$, or $v y$.

2. If $\alpha \beta^{j}$ or $\beta^{j} \gamma$ is covered by one of $x, u, v$, or $y$, then replace this factor by $\alpha \beta$ or $\beta \gamma$, respectively. Otherwise, let us show how to pump when $\alpha \beta^{j} \gamma$ is covered by $x u$. The cases when $\alpha \beta^{j} \gamma$ is covered by $u v$ or $v y$ can be treated analogously. We can factorize $x=x^{\prime} \alpha \beta^{j_{1}} \beta_{1}$ and $u=\beta_{2} \beta^{j_{2}} \gamma u^{\prime}$ where $\beta_{1} \beta_{2}=\beta$ and $j_{1}+j_{2}+1=j$. The pumping results are the words $x^{\prime} \alpha \beta_{1}$ and $\beta_{2} \gamma u^{\prime}$, respectively.

Observe that, after reversing all pumping steps, $\tilde{x} \tilde{u} \sim x u, \tilde{v} \tilde{y} \sim v y, \tilde{x} \tilde{u} \tilde{v} \tilde{y}=x_{1} z y_{1}$, and the rule $t=\left(\tilde{u}, v_{1} ; u_{2}, \tilde{v}\right)$ respects $L$. Furthermore, if we used extension for $u$ (or $\left.v\right)$ in one of the steps, then $|\tilde{u}| \leq m^{2}$ (resp. $|\tilde{v}| \leq m^{2}$ ); in any case $t \in S$. Recall that $w$ was chosen as the shortest word from $L \backslash L(I, S)$. As $\left|\tilde{x} \tilde{u} v_{1}\right|,\left|u_{2} \tilde{v} \tilde{y}\right|<|z|+6 m=|w|$, the words $\tilde{w}_{0}=\tilde{x} \tilde{u} v_{1}$ and $\tilde{w}_{0}^{\prime}=u_{2} \tilde{v} \tilde{y}$ belong to $L(I, S)$, and as $\left(\tilde{w}_{0}, \tilde{w}_{0}^{\prime}\right) \vdash_{t} x_{1} z y_{1}$, we conclude that $x_{1} z y_{1}$ as well as $w$ belong to $L(I, S)-$ the desired contradiction.

Now, we can prove our main result.

Proof of Theorem 4.1. Recall that for a splicing language $L$ with $m=\left|M_{L}\right|$ we intend to prove that the splicing system $(I, R)$ with $I=\Sigma^{<m^{2}+6 m} \cap L$ and

$$
R=\left\{r \in \Sigma^{<m^{2}+10 m} \times \Sigma^{<2 m} \times \Sigma^{<2 m} \times \Sigma^{<m^{2}+10 m} \mid r \text { respects } L\right\}
$$

generates the language $L=L(I, R)$.

Obviously, $L(I, R) \subseteq L$. By Lemma 4.8, we may assume that $L$ is generated by a splicing system $(J, S)$ where

$$
S=\left\{r \in\left(\Sigma^{<m^{2}+10 m}\right)^{4} \mid r \text { respects } L\right\} .
$$

In order to prove $L \subseteq L(I, R)$, we use induction on the length of words in $L$. For $w \in L$ with $|w|<m^{2}+6 m$, by definition, $w \in I \subseteq L(I, R)$.

Now, consider $w \in L$ with $|w| \geq m^{2}+6 m$. The induction hypothesis states that every word $w^{\prime} \in L$ with $\left|w^{\prime}\right|<|w|$ belongs to $L(I, R)$. Factorize $w=x \alpha \beta \gamma \delta y$ such that $|x|=|y|=3 m$, $|\alpha \beta \gamma|=m^{2}, \beta \neq \varepsilon, \alpha \sim \alpha \beta$, and $\gamma \sim \beta \gamma$ (Lemma 2.1).

The proof idea is similar as in the proof of Lemma 4.8. We use a pumping argument on $\beta$ in order to obtain a very long word. This word has to be created by a series of splicings in $(J, S)$. We show that these splicings can be modified in order to create $w$ by splicing from a set of strictly shorter words and with rules from $R$. Then, the induction hypothesis yields $w \in L(I, R)$.

Choose $j$ sufficiently large $\left(j>|w|+m^{2}+10 m\right.$ and $J$ does not contain words of length $j$ or more). We let $z=\alpha \beta^{j} \gamma \delta$ and investigate the creation of $x z y \in L$ by splicing in $(J, S)$. As $z$ is not a factor of a word in $J$, we can trace back the creation of $x z y$ by splicing to the point where the factor $z$ is affected for the last time. Let $z_{k+1}=x_{k+1} z y_{k+1}$, where $x_{k+1}=x$ and $y_{k+1}=y$, be created by $k$ splicings from a word $z_{1}=x_{1} z y_{1}$ which is created by a splicing $\left(w_{0}, w_{0}^{\prime}\right) \vdash_{s} z_{1}$ with $w_{0}, w_{0}^{\prime} \in L, s \in S$, and the splicing position lies in the factor $z$. Furthermore, for $i=1, \ldots, k$ the intermediate splicings are either

(i) $\left(w_{i}, z_{i}\right) \vdash_{r_{i}} x_{i+1} z y_{i+1}=z_{i+1}, w_{i} \in L, r_{i} \in S, y_{i+1}=y_{i}$, and the splicing position lies at the left of the factor $z$ or 
(ii) $\left(z_{i}, w_{i}\right) \vdash_{r_{i}} x_{i+1} z y_{i+1}=z_{i+1}, w_{i} \in L, r_{i} \in S, x_{i+1}=x_{i}$, and the splicing position lies at the right of the factor $z$.

As $|z| \geq m^{2}+10 m$ we can apply Lemma 4.7. Thus, we may assume $w_{1}, \ldots, w_{k} \in I, r_{1}, \ldots, r_{k} \in R$, and $\left|x_{1}\right|,\left|y_{1}\right|<5 m$.

Consider a rule $r_{i}$ in a splicing of the form (i). Suppose the fourth component of $r_{i}$ covers a prefix of the factor $\alpha \beta^{j}$ in $z$ which is longer than $\alpha \beta$ (as $j$ is very large, it cannot fully cover $\alpha \beta^{j}$ ). By extension (Lemma 4.2), we may write $r_{i}=\left(u_{1}, v_{1} ; u_{2}, v^{\prime} \alpha \beta^{\ell}\right)$ for some $\ell \geq 1$. By Lemma 4.3 and as $\alpha \sim \alpha \beta$, we may replace this rule by $\left(u_{1}, v_{1} ; u_{2}, v^{\prime} \alpha\right) \in R$. Moreover, after we symmetrically treated rules of form (ii), these new rules $r_{1}, \ldots, r_{k}$ and the words $w_{1}, \ldots, w_{k}$ can be used in order to create $w=x_{k+1} \alpha \beta \gamma \delta y_{k+1}$ from $x_{1} \alpha \beta \gamma \delta y_{1}$ by splicing. Thus, if $x_{1} \alpha \beta \gamma \delta y_{1}$ belongs to $L(I, R)$, so does $w$.

Now, consider the first splicing $\left(w_{0}, w_{0}^{\prime}\right) \vdash_{s} z_{1}=x_{1} z y_{1}$. By Lemma 4.4 , let $s=\left(u, v_{1} ; u_{2}, v\right)$ such that $w_{0}=x u v_{1}, w_{0}^{\prime}=u_{2} v y$ and $\left|v_{1}\right|,\left|u_{2}\right|<m$ (here, $x$ and $y$ are newly chosen words). Hence,

$$
z_{1}=x u v y=x_{1} z y_{1}=x_{1} \alpha \beta^{j} \gamma \delta y_{1}
$$

where $x u$ is a proper prefix of $x_{1} z$ and $v y$ is a proper suffix of $z y_{1}$.

Next, we will pump down the factor $\alpha \beta^{j} \gamma$ to $\alpha \beta \gamma$ in $z$ again in order to obtain the words $\tilde{x}, \tilde{u}, \tilde{v}, \tilde{y}$ from the word $x, u, v, y$, respectively. The pumping is done as in the proof of Lemma 4.8:

1. If $u$ is covered by the factor $\alpha \beta^{j} \gamma$, extend $u$ to the left such that it becomes a prefix of $\alpha \beta^{j} \gamma$. Symmetrically, if $v$ is covered by the factor $\alpha \beta^{j} \gamma$, extend $v$ to the right such that it becomes a suffix of $\alpha \beta^{j} \gamma$ (Lemma 4.2). Observe that extension ensures that the factor $\alpha \beta^{j} \gamma$ is covered by either $x u, u v$, or $v y$.

2. If $\alpha \beta^{j}$ or $\beta^{j} \gamma$ is covered by one of $x, u, v$, or $y$, then replace this factor by $\alpha \beta$ or $\beta \gamma$, respectively. Otherwise, let us show how to pump when $\alpha \beta^{j} \gamma$ is covered by $x u$. The cases when $\alpha \beta^{j} \gamma$ is covered by $u v$ or $v y$ can be treated analogously. We can factorize $x=x^{\prime} \alpha \beta^{j_{1}} \beta_{1}$ and $u=\beta_{2} \beta^{j_{2}} \gamma u^{\prime}$ where $\beta_{1} \beta_{2}=\beta$ and $j_{1}+j_{2}+1=j$. The pumping result are the words $x^{\prime} \alpha \beta_{1}$ and $\beta_{2} \gamma u^{\prime}$, respectively.

Observe that, $\tilde{x} \tilde{u} \sim x u, \tilde{v} \tilde{y} \sim v y, \tilde{x} \tilde{u} \tilde{v} \tilde{y}=x_{1} \alpha \beta \gamma \delta y_{1}$, and the rule $t=\left(\tilde{u}, v_{1} ; u_{2}, \tilde{v}\right)$ respects $L$. Furthermore, if we used extension for $u$ (or $v$ ), then $|\tilde{u}| \leq m^{2}$ (resp. $|\tilde{v}| \leq m^{2}$ ). No matter whether we used extension, $t \in R$. As $\left|\tilde{x} \tilde{u} v_{1}\right|,\left|u_{2} \tilde{v} \tilde{y}\right|<|z|+6 m=|w|$ and by induction hypothesis, the words $\tilde{w}_{0}=\tilde{x} \tilde{u} v_{1}$ and $\tilde{w}_{0}=u_{2} \tilde{v} \tilde{y}$ belong to $L(I, S)$. We conclude that $\left(\tilde{w}_{0}, \tilde{w}_{0}^{\prime}\right) \vdash_{t} x_{1} \alpha \beta \gamma \delta y_{1} \in$ $L(I, R)$ and, therefore, $w=x_{k+1} \alpha \beta \gamma \delta y_{k+1} \in L(I, R)$ as well.

\section{Decidability}

The main question we intended to answer when starting our investigation was, whether or not it is decidable if a given regular language $L$ is a splicing language. If we can decide whether a splicing rule respects a regular language and if we can construct a (non-deterministic) finite automaton accepting the language generated by a given splicing system, then we can decide whether $L$ is a classic splicing language (Pixton splicing language) as follows. We compute the splicing system $(I, R)$ as given in Theorem 4.1 (resp. Theorem 3.1) and we compute a finite automaton accepting the splicing language $L(I, R)$. Theorem 4.1 (resp. Theorem 3.1) implies that $L$ is a splicing language if and only if $L=L(I, R)$. Recall that equivalence of regular languages is decidable, e. g., by constructing and comparing the minimal deterministic finite automata of both languages.

It is known from $[8,13]$ that it is decidable whether a classic splicing rule respects a regular language. Furthermore, there is an effective construction of a finite automaton which accepts the language generated by a Pixton splicing system [17]. As mentioned earlier, Pixton splicing systems are more general than classic splicing systems, which means the latter result applies to classic splicing systems, too. Such a construction for classic splicing systems is also given in [12].

Let us prove that it is decidable whether a Pixton splicing rule $r$ respects a regular language $L$. Actually, we will decide whether the set $[r]_{L}$ respects $L$, which is equivalent by Lemma 4.3. 
The proof can easily be adapted in order to prove that it is decidable whether a classic splicing rule respects $L$.

Lemma 5.1. Let $L$ be a regular language and let $r$ be a Pixton splicing rule. It is decidable whether $r$ respects $L$.

Proof. Let $\sim$ denote the equivalence relation $\sim_{L}$ and $[\cdot]$ denote the corresponding equivalence classes $[\cdot]_{L}$.

Let $r=\left(u_{1}, u_{2} ; v\right)$. We define the two sets $S_{1}, S_{2} \subseteq M_{L}$ as

$$
S_{1}=\left\{X \in M_{L} \mid \exists Y: X\left[u_{1}\right] Y \subseteq L\right\}, \quad S_{2}=\left\{Y \in M_{L} \mid \exists X: X\left[u_{2}\right] Y \subseteq L\right\},
$$

i. e., $\left[x_{1}\right]$ belongs to $S_{1}$ if and only if $x_{1} u_{1} y_{1} \in L$ for some word $y_{1}$ and $\left[y_{2}\right]$ belongs to $S_{2}$ if and only if $x_{2} u_{2} y_{2} \in L$ for some word $x_{2}$. We claim that $r$ respects $L$ if and only if $X[v] Y \subseteq L$ for all $X \in S_{1}$ and $Y \in S_{2}$, which is a property that can easily be decided.

Firstly, suppose $r$ respects $L$. For $X \in S_{1}$ and $Y \in S_{2}$ choose words $x_{1} \in X$ and $y_{2} \in Y$. By definition of $S_{1}$ and $S_{2}$, there is $y_{1}$ and $x_{2}$ such that $x_{i} u_{i} y_{i} \in L$ for $i=1,2$ and, as $r$ respects $L$, $x_{1} v y_{2} \in L$. This implies $X[v] Y \subseteq L$.

Vice verse, suppose $X[v] Y \subseteq L$ for all $X \in S_{1}$ and $Y \in S_{2}$. For all $x_{i} u_{i} y_{i} \in L$ with $i=1,2$, we have $\left[x_{1}\right] \in S_{1}$ and $\left[y_{2}\right] \in S_{2}$. Therefore, $x_{1} v y_{2} \in\left[x_{1}\right][v]\left[y_{2}\right] \subseteq L$ and $r$ respects $L$.

These observations lead to the decidability results.

\section{Corollary 5.2.}

i.) For a given regular language $L$, it is decidable whether or not $L$ is a classic splicing language. Moreover, if $L$ is a classic splicing language, a splicing system $(I, R)$ generating $L$ can be effectively constructed.

ii.) For a given regular language $L$, it is decidable whether or not $L$ is a Pixton splicing language. Moreover, if $L$ is a Pixton splicing language, a splicing system $(I, R)$ generating $L$ can be effectively constructed.

\section{Final Remarks}

It has been known since 1991 that the class $\mathcal{S}$ of languages that can be generated by a splicing system is a proper subclass of the class of regular languages. However, to date, no other natural characterization for the class $\mathcal{S}$ exists. The problem of deciding whether a regular language is generated by a splicing system is a fundamental problem in this context and has remained unsolved. To the best of our knowledge, the problem was first stated in the literature in 1998 [11]. In this paper we solved this long standing open problem.

Regarding the complexity of the decision algorithm, let $L$ be a regular language given as syntactic monoid $M_{L}$ and $(I, R)$ be the splicing system described in Theorem 4.1 (resp. Theorem 3.1). An automaton which accepts $L(I, R)$ and is created as described in Section 5 has a state set of size in $2^{\mathcal{O}\left(m^{2}\right)}$, where $m=\left|M_{L}\right|$. Deciding the equivalence of two regular languages, given as NFAs, is known to be PSPACE-complete [20]; hence, the naive approach to decide whether or not $L=L(I, R)$ uses double exponential time $2^{2^{\mathcal{O}\left(m^{2}\right)}}$. As there may be an exponential gap between an NFA accepting $L$ and the syntactic monoid $M_{L}$, the complexity, when considering an NFA as input, becomes triple exponential. Improving the complexity of the algorithm is subject of future research.

\section{References}

[1] P. Bonizzoni. Constants and label-equivalence: A decision procedure for reflexive regular splicing languages. Theor. Comput. Sci., 411(6):865-877, 2010. 
[2] P. Bonizzoni, C. de Felice, and R. Zizza. The structure of reflexive regular splicing languages via Schützenberger constants. Theor. Comput. Sci., 334(1-3):71-98, 2005.

[3] P. Bonizzoni, C. de Felice, and R. Zizza. A characterization of (regular) circular languages generated by monotone complete splicing systems. Theor. Comput. Sci., 411(48):4149-4161, 2010 .

[4] P. Bonizzoni, C. Ferretti, G. Mauri, and R. Zizza. Separating some splicing models. Inf. Process. Lett., 79(6):255-259, 2001.

[5] P. Bonizzoni and N. Jonoska. Regular splicing languages must have a constant. In G. Mauri and A. Leporati, editors, Developments in Language Theory, volume 6795 of Lecture Notes in Computer Science, pages 82-92. Springer Berlin / Heidelberg, 2011.

[6] K. Culik II and T. Harju. Splicing semigroups of dominoes and DNA. Discrete Applied Mathematics, 31(3):261-277, 1991.

[7] R. W. Gatterdam. Splicing systems and regularity. International Journal of Computer Mathematics, 31(1-2):63-67, 1989.

[8] E. Goode. Constants and Splicing Systems. PhD thesis, Binghamton University, 1999.

[9] E. Goode and D. Pixton. Recognizing splicing languages: Syntactic monoids and simultaneous pumping. Discrete Applied Mathematics, 155(8):989-1006, 2007.

[10] T. Head. Formal language theory and DNA: an analysis of the generative capacity of specific recombinant behaviors. Bulletin of Mathematical Biology, 49(6):737-759, 1987.

[11] T. Head. Splicing languages generated with one sided context. In G. Păun, editor, Computing With Bio-molecules: Theory and Experiments, pages 269-282. Springer Verlag, 1998.

[12] T. Head and D. Pixton. Splicing and regularity. In Z. Ésik, C. Martín-Vide, and V. Mitrana, editors, Recent Advances in Formal Languages and Applications, volume 25 of Studies in Computational Intelligence, pages 119-147. Springer, 2006.

[13] T. Head, D. Pixton, and E. Goode. Splicing systems: Regularity and below. In M. Hagiya and A. Ohuchi, editors, DNA, volume 2568 of Lecture Notes in Computer Science, pages 262-268. Springer, 2002.

[14] J. E. Hopcroft and J. D. Ullman. Introduction to Automata Theory, Languages and Computation. Addison-Wesley, 1979.

[15] L. Kari and S. Kopecki. Deciding whether a regular language is generated by a splicing system. In D. Stefanovic and A. Turberfield, editors, DNA, volume 7433 of Lecture Notes in Computer Science, pages 98-109. Springer, 2012.

[16] S. M. Kim. An algorithm for identifying spliced languages. In T. Jiang and D. T. Lee, editors, COCOON, volume 1276 of Lecture Notes in Computer Science, pages 403-411. Springer, 1997.

[17] D. Pixton. Regularity of splicing languages. Discrete Applied Mathematics, 69(1-2):101-124, 1996.

[18] G. Păun. On the splicing operation. Discrete Applied Mathematics, 70(1):57 - 79, 1996.

[19] M. P. Schützenberger. Sur certaines opérations de fermeture dans le langages rationnels. Symposia Mathematica, 15:245-253, 1975.

[20] L. J. Stockmeyer and A. R. Meyer. Word problems requiring exponential time: Preliminary report. In A. V. Aho, A. Borodin, R. L. Constable, R. W. Floyd, M. A. Harrison, R. M. Karp, and H. R. Strong, editors, STOC, pages 1-9. ACM, 1973. 